\title{
TMK-based cell-surface auxin signalling activates cell-wall acidification
}

https://doi.org/10.1038/s41586-021-03976-4

Received: 3 February 2021

Accepted: 31 August 2021

Published online: 27 October 2021

\section{Open access}

Check for updates

\author{
Wenwei Lin ${ }^{1,2}$, Xiang Zhou ${ }^{1,2}$, Wenxin Tang', Koji Takahashi ${ }^{3,4}$, Xue Pan ${ }^{2}$, Jiawei Dai', Hong Ren ${ }^{5}$, \\ Xiaoyue Zhu', Songqin Pan ${ }^{2}$, Haiyan Zheng ${ }^{6}$, William M. Gray ${ }^{5}$, Tongda Xu', \\ Toshinori Kinoshita ${ }^{3,4}$ \& Zhenbiao Yang ${ }^{1,2 \bowtie}$
}

\begin{abstract}
The phytohormone auxin controls many processes in plants, at least in part through its regulation of cell expansion ${ }^{1}$. The acid growth hypothesis has been proposed to explain auxin-stimulated cell expansion for five decades, but the mechanism that underlies auxin-induced cell-wall acidification is poorly characterized. Auxin induces the phosphorylation and activation of the plasma membrane $\mathrm{H}^{+}$-ATPase that pumps protons into the apoplast ${ }^{2}$, yet how auxin activates its phosphorylation remains unclear. Here we show that the transmembrane kinase (TMK) auxin-signalling proteins interact with plasma membrane $\mathrm{H}^{+}$-ATPases, inducing their phosphorylation, and thereby promoting cell-wall acidification and hypocotyl cell elongation in Arabidopsis. Auxin induced interactions between TMKs and $\mathrm{H}^{+}$-ATPases in the plasma membrane within seconds, as well as TMK-dependent phosphorylation of the penultimate threonine residue on the $\mathrm{H}+$-ATPases. Our genetic, biochemical and molecular evidence demonstrates that TMKs directly phosphorylate plasma membrane $\mathrm{H}^{+}$-ATPase and are required for auxin-induced $\mathrm{H}^{+}$-ATPase activation, apoplastic acidification and cell expansion. Thus, our findings reveal a crucial connection between auxin and plasma membrane $\mathrm{H}^{+}$-ATPase activation in regulating apoplastic $\mathrm{pH}$ changes and cell expansion through TMK-based cell surface auxin signalling.
\end{abstract}

Embedded in a rigid cell wall, the plant cell must modify its wall to gain the adjustable elasticity to regulate cell expansion in space and time. Auxin induces rapid cell expansion by acidifying the cell-wall space (apoplast), leading to the activation of cell-wall-localized proteins for wall loosening ${ }^{3,4}$, a growth mechanism that has been known as the acid growth theory for half of a century ${ }^{5}$. Auxin triggers the efflux of protons, resulting in apoplastic acidification by activating the plasma membrane (PM)-localized P-type $\mathrm{H}^{+}$-ATPase ${ }^{6,7}$. In Arabidopsis, $\mathrm{PM} \mathrm{H}^{+}$-ATPase is encoded by an autoinhibited $\mathrm{H}^{+}$-ATPase (AHA) gene family comprising 11 members ${ }^{8}$. Phosphorylation of the conserved penultimate $\mathrm{Thr}$ residue (Thr948 in AHA1, Thr947 in AHA2) has been proposed to release the autoinhibition of the ATPase pump activity by the cytoplasmic C-terminal region ${ }^{9-15}$. Fendrych et al. previously demonstrated that auxin-induced apoplastic acidification and growth are mediated by TIR1/AFB-Aux/IAA nuclear auxin perception in hypocotyls ${ }^{16}$. Auxin induces the TIR1/AFB-dependent expression of SAUR proteins that act as inhibitors of PP2C.D phosphatases, which dephosphorylate the penultimate $\mathrm{Thr}$ residue ${ }^{17}$. Although this mechanism can sustain $\mathrm{H}^{+}$-ATPase activity by preventing the dephosphorylation of the penultimate $\mathrm{Thr}$ residue, it cannot account for how the $\mathrm{PM} \mathrm{H}^{+}$-ATPase is initially phosphorylated to become activated.

The PM-localized TMK-receptor-like kinases have a vital role in auxin signalling in regulating pavement cell morphogenesis, differential growth of the apical hook, lateral root formation and root thermomorphogenesis in Arabidopsis $^{18-23}$. Auxin rapidly promotes TMK-dependent activation of PM-associated ROP GTPases within seconds, providing a mechanism for rapid auxin responses on the cell surface in addition to TIR1/AFB-based intracellular auxin signalling ${ }^{20,24-26}$. To identify new components in TMK-mediated auxin-signalling pathways, we performed immunoprecipitation coupled with mass spectrometry (IP-MS) to isolate potential interactors of TMK1 in Arabidopsis. In brief, GFP-Trap agarose beads were used to immunoprecipitate the TMK1-GFP protein complex from $p T M K 1:: T M K 1-G F P$ transgenic plants, which was further analysed using MS. The proteins that were identified only from the $p T M K 1:: T M K 1-G F P$ transgenic plants but not from the $p T M K 1:: G F P$ control plants were considered to be candidates for TMK1-associated proteins (Supplementary Table 2). Among them, we were especially interested in the $\mathrm{PM} \mathrm{H}^{+}$-ATPases (AHAs) (Extended Data Fig. 1a), as the previous study showed that auxin triggers the activation of the $\mathrm{PM} \mathrm{H}^{+}$-ATPase, which promotes hypocotyl cell elongation ${ }^{27}$. We further confirmed that GFP-AHA1 co-immunoprecipitated with TMK1 and TMK4 in the 35S::GFP-AHA1 transgenic plants as detected by immunoblotting using anti-TMK1 and anti-TMK4 antibodies, respectively (Fig. 1a and Extended Data Fig. 1b). Furthermore, TMK1-GFP co-immunoprecipitated with $\mathrm{AHA}(\mathrm{s})$ from $p T M K 1:$ :TMK1-GFP transgenic plants as detected by immunoblot analysis using anti-AHA2-cat

${ }^{1}$ FAFU-UCR Joint Center for Horticultural Biology and Metabolomics, Haixia Institute of Science and Technology, Fujian Agriculture and Forestry University, Fuzhou, China. ${ }^{2}$ Institute of Integrative Genome Biology and Department of Botany and Plant Science, University of California, Riverside, CA, USA. ${ }^{3}$ Graduate School of Science, Nagoya University, Nagoya, Japan. ${ }^{4}$ Institute of Transformative Bio-Molecules, Nagoya University, Nagoya, Japan. ${ }^{5}$ Department of Plant and Microbial Biology, University of Minnesota, St Paul, MN, USA. ${ }^{6}$ Biological Mass Spectrometry Facility, Robert Wood Johnson Medical School and Rutgers, the State University of New Jersey, Piscataway, NJ, USA. ${ }^{凶}$-mail: yang@ucr.edu 


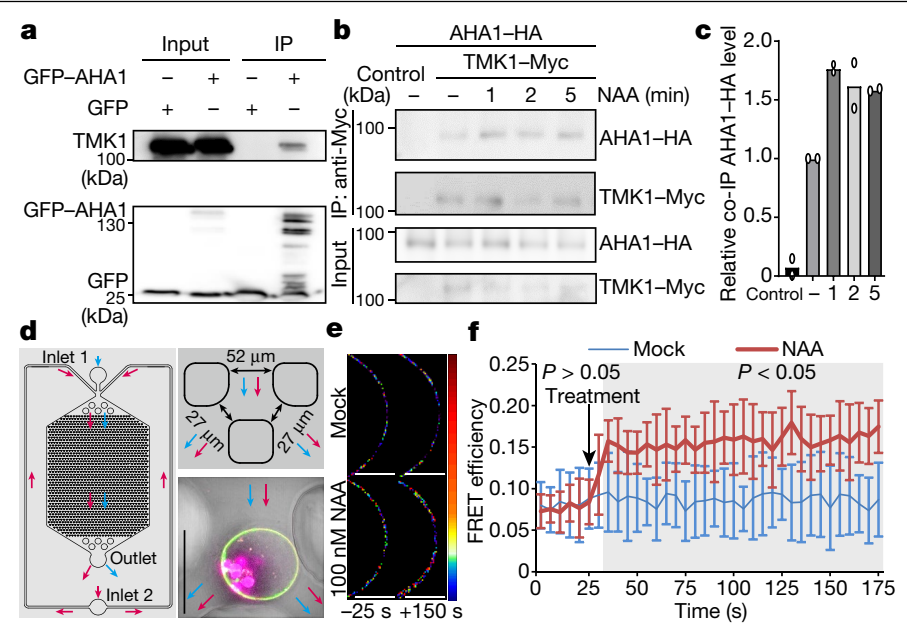

Fig. 1 TMK1 interacts directly with AHAs. a, Co-IP analysis of TMK1 with GFPAHA1.35S::GFP (control) and 35S::GFP-AHA1 plants were immunoprecipitated with anti-GFP antibodies and analysed by western blotting using anti-TMK1 antibodies. b, Auxin induced interactions between TMK1-Myc and AHA-HA in Arabidopsis protoplasts. AHA1-HA and TMK1-Myc constructs were transiently expressed in protoplasts, which were then treated with $1 \mu$ MNAA for 1,2 and 5 min before being used for co-IP analysis. c, Quantification of AHA1-HA proteins co-immunoprecipitated with TMK1-Myc as shown in b. Data are the mean values of two independent biological replicates. d, The microfluidics device that was designed to investigate the auxin-induced rapid TMK1-AHA1 interaction using FRET analysis. Right, a triangle trap (top) for trapping a protoplast (bottom). The blue arrows indicate the flow of cell suspension, and the red arrows indicate the flow of NAA or mock solutions. Scale bar, $50 \mu \mathrm{m}$. e, FRET analysis of the rapid induction of the TMK1-AHA interaction. A representative heat map of sensitized emission efficiencies for the TMK1-mCherry/AHA1-GFPFRET on the PM region (further details are provided in Extended Data Fig. 1d). The times before and after treatment are indicated $(-25 \mathrm{~s}$ and $+150 \mathrm{~s})$. Scale bars, $10 \mu \mathrm{m}$.f, Quantitative time-course analyses of changes in the FRET efficiencies. NAA $(100 \mathrm{nM})$ or mock buffer was applied at $25 \mathrm{~s}$ after imaging started. The error bars indicate the s.d. of 10 cells scored. Statistical analysis was performed using two-sided Student's $t$-tests $(P<0.05)$; the grey background indicates significant differences during the covered periods.

antibodies after anti-GFP-Trap antibody immunoprecipitation (Extended Data Fig. 1c). An in vitro pull-down assay showed that the kinase domain of TMK1 (TMK1 ${ }^{\mathrm{KD}}$ ), when fused to maltose-binding protein (MBP), directly interacted with the AHA2 C-terminal domain fused to glutathione $S$-transferase (GST) (GST-AHA2-C) (Extended Data Fig. 1d), suggesting that the kinase domain of TMK1 directly binds to the $\mathrm{C}$-terminal region of AHA2. We postulated that TMK1 interacts with AHAs in vivo as both proteins are predominantly localized at the $\mathrm{PM}^{20,28,29}$. A co-immunoprecipitation (co-IP) assay showed that the in vivo association between Myc-tagged TMK1 and HA-tagged AHA1 was enhanced within 1 min after treatment with 1-naphthaleneacetic acid (NAA) (Fig. 1b, c), suggesting that auxin rapidly promotes TMK1-AHA interactions. To test whether auxin rapidly induces a direct interaction between TMK1 and AHA1, we developed a microfluidics device enabling the high-resolution time-course analysis of a rapid induction of the dynamic interaction in a single Arabidopsis protoplast using fluorescence resonance energy transfer (FRET) imaging (Fig. 1d). Protoplasts co-expressing TMK1-mCherry and AHA1-GFP were captured in triangle traps within the device, enabling imaging before and immediately after auxin treatment (Fig.1d and Extended Data Fig. 1e). Intriguingly, the TMK1-mCherry/AHA1-GFP FRET efficiency increased within $10 \mathrm{~s}$ after auxin treatment, indicating that auxin very rapidly promotes the direct interaction between TMK1-mCherry and AHA1-GFP (Fig. 1e,f). By contrast, no increase in FRET efficiency was detected when protoplasts co-expressing TMK1-mCherry and AHA1-GFP were mock-treated with control buffer (Fig. 1e,f). Together, these results show that auxin promotes a rapid and direct interaction between TMK1 and AHA1 on the PM.

The phosphorylation of the conserved penultimate threonine residue on the $\mathrm{H}^{+}$-ATPase proteins is a primary mechanism by which the $\mathrm{H}^{+}$-ATPase is activated in response to multiple signals, including phytohormones, sucrose, $\mathrm{NaCl}$, blue light and the fungal toxin fusico$\operatorname{ccin}^{13,14,27,30-32}$. We first examined the phosphorylation status of the penultimate threonine residue in the aerial parts of Arabidopsis seedlings using phosphoproteomics (with the roots removed when the seedlings were prepared for the assay). The phosphorylation levels of the penultimate Thr residue of AHA2, AHA3 and AHA7 were compromised in the tmk1-1 tmk4-1 mutant compared with the wild type (Extended Data Fig. 2a-e), implying a general reduction of $\mathrm{H}^{+}$-ATPase activity in the mutant. TMK1 and TMK 4 are functionally redundant in the regulation of the growth of Arabidopsis seedlings, as neither of the tmk 1 and $t m k 4$ single-knockout mutants exhibit a visible growth defect, whereas the tmk1 tmk4 double mutants show severe growth retardation, especially in hypocotyl elongation ${ }^{28}$ (also see below).

We next analysed the phosphorylation status of the penultimate Thr residue by immunoblotting using antibodies against phosphorylated Thr947 (pThr947), which recognize the unique phosphorylation of the penultimate Thr residue in all of the AHA isoforms ${ }^{27}$. Fusicoccin promotes the binding of 14-3-3 to the phosphorylated C-terminal region of $\mathrm{PM} \mathrm{H}^{+}$-ATPase, resulting in the activation of the pump. As shown previously ${ }^{14}$, fusicoccin increased the level of phosphorylation of the penultimate Thr residue in wild type Col-0 seedlings (Extended Data Fig. 2f, g). Similarly, treatments with auxin at micromolar or nanomolar levels increased its phosphorylation levels (Fig. 2a, b and Extended Data Fig. 2f, g). Compared with the untreated wild type, the level of phosphorylation of the penultimate Thr residue was reduced in the tmk1-1 tmk4-1 mutant (Fig. 2a, b and Extended Data Fig. 2f, g). Importantly, auxin-induced phosphorylation of this residue was nearly abolished in the tmk1-1 tmk4-1 mutant (Fig. 2a, b and Extended Data Fig. 2f, g). By contrast, fusicoccin treatment still increased the level of phosphorylation of the penultimate Thr residue in the tmk1-1 tmk4-1 mutant (Extended Data Fig. 2f, g), suggesting that the tmk1-1 tmk4-1 mutant was able to respond to other stimuli in regulating AHA phosphorylation at the penultimate Thr residue. Thus, TMK1 and TMK4 are required selectively for the auxin-induced increase in phosphorylation of the penultimate Thr residue.

To assess whether TMKs directly phosphorylate AHA at this penultimate Thr residue, we next immunoprecipitated AHA1-GFP from Arabidopsis protoplasts that transiently expressed this fusion protein for an in vitro phosphorylation assay. Recombinant TMK1 kinase domain $\left(\mathrm{TMK} 1^{\mathrm{KD}}\right)$, but not thekinase-deadmutant $\left(\mathrm{TMK} 1^{\mathrm{Km}}\right)$, greatlyincreased the phosphorylation of AHA1-GFP at the Thr948 residue in vitro (Fig. 2c, d). We further determined whether TMK1 phosphorylates a synthetic peptide containing the $16 \mathrm{C}$-terminal amino acid residues from AHA1 (AHA1-C16) in an in vitro assay using the recombinant TMK1 ${ }^{\mathrm{KD}}$. MS analysis showed that the penultimate Thr residue ( $\mathrm{Thr} 15$ of the peptide, Thr948 of AHA1) of AHA1-C16 was highly phosphorylated by TMK1 ${ }^{\mathrm{KD}}$, but not by TMK1 ${ }^{\mathrm{Km}}$ (Fig. 2e and Extended Data Fig. $2 \mathrm{~h}$ ). The second to the last Thr residue (T9T15, Thr9 and Thr15 in the AHA1-C16 peptide.) on AHA1-C16 was weakly phosphorylated by TMK $1^{\mathrm{KD}}$. Neither TMK $1^{\mathrm{KD}}$ nor TMK $1^{\mathrm{Km}}$ phosphorylated a scrambled synthetic peptide (Fig. 2e). Thus, TMK1 specifically phosphorylates the penultimate Thr residue of AHA1. Together with auxin-induced rapid interaction between TMK1 and AHA1 and the requirement of TMK1 and TMK4 for auxin-induced AHA phosphorylation in vivo, these results strongly indicate a role for auxin-activated TMK1 in directly phosphorylating AHA1.

We next investigated whether TMK1 and TMK4 are required for the activation of $\mathrm{PM} \mathrm{H}^{+}$-ATPase by auxin. The activation of $\mathrm{PM} \mathrm{H}^{+}$-ATPase couples with the ATP hydrolysis ${ }^{14}$. As shown previously ${ }^{27}$, auxin treatment for 30 min increased ATP hydrolysis in the aerial parts of wild-type Arabidopsis seedlings by 50\% (Fig. 2f). Neither tmk1-1 nor 


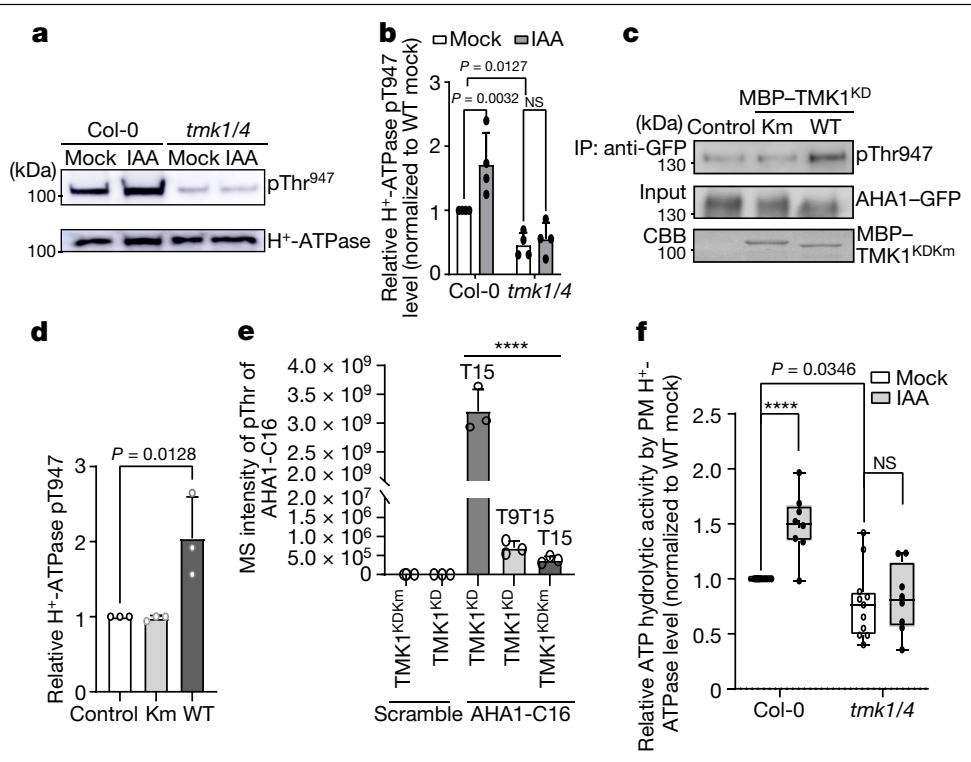

Fig. 2 | TMK1 and TMK4 are required for auxin-induced phosphorylation and activation of the $\mathrm{PM} \mathrm{H}^{+}$-ATPase. a, Western blot detection of phosphorylated $\mathrm{H}^{+}$-ATPase in the aerial parts of wild-type and tmk1-1 tmk4-1 (tmk1/4) mutant plants (top, anti-pThr947). AHA protein levels were determined using anti- $\mathrm{H}^{+}$-ATPase antibodies (bottom). Seedlings were treated with mock buffer or $10 \mu$ M IAA for 30 min. b, Quantification of the phosphorylation level of the $\mathrm{H}^{+}$-ATPase. Data are mean \pm s.d. $n=4$ independent experiments.c, TMK1 ${ }^{\mathrm{KD}}$ phosphorylated AHA1-GFP in vitro. TMK1 (TMK1 ${ }^{\mathrm{KD}}, \mathrm{WT}$ ) or the kinase-dead form (TMK1 $1^{\mathrm{KDKm}}$ ) was incubated with protoplast-expressed AHA1-GFP, and its phosphorylation state was determined using anti-pThr947 (pThr947) antibodies. CBB, Coomassie Brilliant Blue. d, Quantification of the Thr948 phosphorylation level (determined using anti-pThr947 antibodies) of the AHA1-GFP. Data are mean \pm s.d. $n=3$ biological replicates. e, MS detection of AHA1-C16 phosphorylation by TMK ${ }^{\mathrm{KD}}$ in vitro. The graph shows the abundance of phosphorylated peptides at the indicated residues analysed by MS. Data are mean \pm s.d. $n=3$. Two biological replicates with three technical replicates each were performed.f, Auxin induction of $\mathrm{H}^{+}$-ATPase activity was abolished in tmk1-1 tmk4-1. The values shown are relative ATP hydrolytic activity of indicated samples to that of mock Col-0. The box bounds the interquartile range divided by the median (central lines), and the Tukey-style whiskers extend to a maximum of $1.5 \times$ interquartile range from 25 th and 75 th percentiles. $n=11$ (mock) and $n=8$ (IAA). Statistical analysis was performed using two-way ANOVA (b and $\mathbf{f})$ or one-way ANOVA ( $\mathbf{d}$ and $\mathbf{e}) ;{ }^{* * * *} P<0.0001$. tmk4-1 mutations significantly affected the basal level of ATP hydrolysis or auxin-induced changes in ATP hydrolysis (Extended Data Fig. 2i). However, the basal level of ATP hydrolysis was significantly reduced in the tmk1-1 tmk4-1 mutant (Fig. 2f), consistent with the reduced level of phosphorylation of the penultimate Thr residue (Fig. 2a, b). Importantly, auxin-induced enhancement of ATP hydrolysis was abolished in this double mutant (Fig. 2f), indicating that TMK1 and TMK4 are essential for auxin-induced $\mathrm{H}^{+}$-ATPase activation. In agreement with the compromised $\mathrm{H}^{+}$-ATPase activity, compared with the wild type, the tmk1-1 tmk4-1 mutant was more tolerant to lithium (Extended Data Fig. $2 \mathrm{j}, \mathrm{k}$ ), a toxic alkali cation, the uptake of which is coupled with the activation of $\mathrm{H}^{+}$-ATPase and $\mathrm{PM}$ hyperpolarization. In particular, the aerial part of wild-type seedlings became chlorotic, whereas the aerial part of the tmk1-1 tmk4-1 seedlings remained green after growth on lithium. This is in contrast to SAUR19-OX lines, which display increased $\mathrm{H}^{+}$-ATPase activity and therefore exhibit much higher sensitivity to lithium ${ }^{17}$. These findings together demonstrate that TMK1 and TMK4 are required for auxin-induced $\mathrm{PM}^{+}$-ATPase activation.

To assess the consequences of the reduced $\mathrm{PM} \mathrm{H}^{+}$-ATPase activation in tmk1-1 tmk4-1 plants, we introduced membrane-impermeable 8-hydroxypyrene-1,3,6-trisulfonic acid trisodium salt (HPTS) as a ratiometric fluorescent $\mathrm{pH}$ indicator for assessing changes in the apoplastic $\mathrm{pH}$ at a cellular resolution in Arabidopsis thaliana hypocotyls ${ }^{4}$. Two different forms of HPTS (the protonated and deprotonated forms) were visualized in two independent channels with excitation wavelengths of $405 \mathrm{~nm}$ and $458 \mathrm{~nm}$, respectively. The apoplastic $\mathrm{pH}$ correlates with the ratiometric values (signal intensity from the $458 \mathrm{~nm}$ channel divided by that from the $405 \mathrm{~nm}$ channel $)^{4,33}$. As a positive control for the HPTS-based $\mathrm{pH}$ indicator, we monitored the apoplastic $\mathrm{pH}$ in hypocotyls of the ost $2-2 D$ mutant harbouring the constitutively activated AHA1 (ref. ${ }^{34}$ ). As shown previously ${ }^{4}$, the ost $2-2 D$ mutant exhibited lower 458/405 values compared with the wild type (Fig. 3a, b), confirming its enhanced apoplastic acidification. By contrast, significantly higher 458/405 values were observed in tmk1-1 tmk4-1 hypocotyls, suggesting apoplastic alkalization in the mutant (Fig. 3a, b). Furthermore, the apoplastic $\mathrm{pH}$ of the tmk1-1 tmk4-1 mutant was restored to the wild-type level when this mutant was complemented with wild-type TMK1 (Extended Data Fig. 3a, b), indicating that TMK1 is essential for the regulation of the apoplastic $\mathrm{pH}$.

Importantly, hypocotyl cell length was correlated with the $\mathrm{pH}$ value of the mutant when compared with the wild type (Fig. 3c). In tmk1-1 tmk4-1 mutants, the mean length of hypocotyl cells was significantly shorter than in the wild type ${ }^{28}$ (Figs. 3a, c), and the hypocotyl cell lengths were

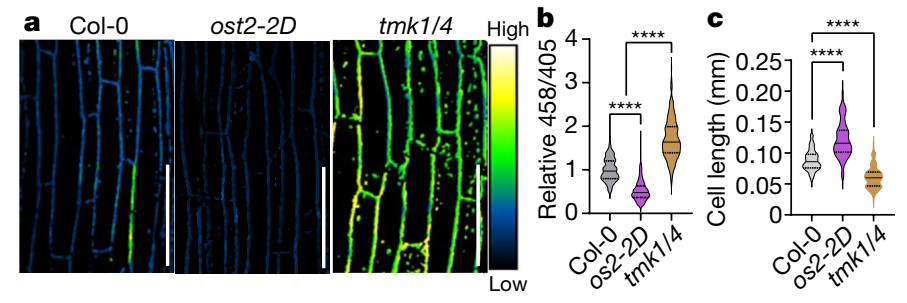

Fig. 3 | TMK1 and TMK4 are required for apoplastic acidification and cell elongation in Arabidopsis hypocotyl. a, b, Comparison (imaging (a) and quantification (b)) of the apoplastic $\mathrm{pH}$ in wild type (Col-0), ost2-2D and tmk1-1 tmk4-1 plants. Changes in $\mathrm{pH}$ were visualized with ratiometric values of fluorescent HPTS. The mean 458/405 values of ost2-2D and the tmk1-1 tmk4-1 mutant relative to the WT are shown on the $y$ axis. $n=300$ ( 6 hypocotyls, 50 cells for each). c, Epidermal cell lengths of hypocotyls. $n=140$ (Col-0), $n=64$ (ost2-2D) and $n=134$ (tmk1-1 tmk4-1) cells. Statistical analysis was performed using one-way ANOVA (b and $\mathbf{c}$ ) (three independent assays); ${ }^{* * * *} P \leq 0.0001$. For a, scale bars, $100 \mu \mathrm{m}$. 


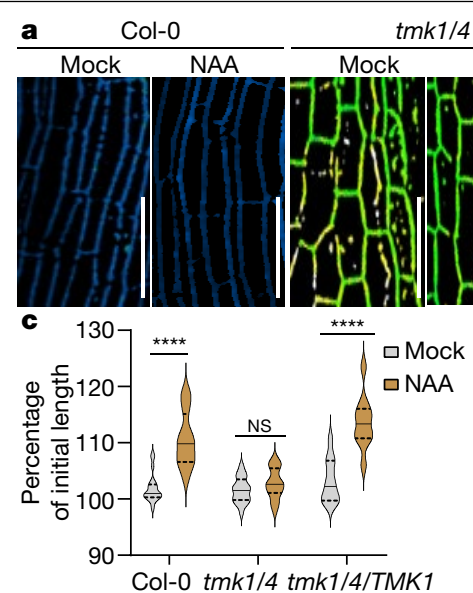

Fig. 4 | TMK1 and TMK4 are required for auxin-induced apoplastic acidification and hypocotyl elongation. a, The effect of auxin (100 nMNAA for $15 \mathrm{~min}$ ) on the apoplastic $\mathrm{pH}$ changes visualized by HPTS staining. b, Quantitative analysis of a. $n=350$ ( 7 hypocotyls, 50 cells for each). c, Auxin-induced rapid elongation hypocotyl segments from wild-type, $t m k 1-1 \mathrm{tmk} 4-1(\mathrm{tmk} 1 / 4)$ and tmk1-1 tmk4-1 TMK1 (tmk1/4/TMK1) plants. The relative length when comparing

largely restored when the mutant was complemented with wild-type TMK1 (Extended Data Fig. 3c). By contrast, increased apoplastic acidification is linked to an increase in cell length and hypocotyl length in ost2-2D plants (Fig. 3a, c and Extended Data Fig. 3d, e).

Auxin promotes apoplastic acidification in hypocotyls through the activation of $\mathrm{PM} \mathrm{H}^{+}$-ATPase, contributing to auxin-induced cell elongation $^{16,27}$. We found that the auxin-induced acidification in the apoplast was abolished in tmk1-1 tmk4-1 plants (Fig. 4a, b), suggesting an essential role of TMK1 and TMK4 in auxin-triggered $\mathrm{PM} \mathrm{H}^{+}$-ATPase activation. Moreover, exogenous NAA promoted the rapid elongation of auxin-depleted hypocotyl segments (Fig. 4c) and the elongation of whole hypocotyls (Fig. 4d) from wild-type seedlings, but not from the tmk1-1 tmk4-1 seedlings. The tmk1-1 tmk4-1 mutant complemented with TMK1 exhibited a normal response to auxin in promoting hypocotyl segment elongation (Fig. 4c). The severe defect in tmk1-1 tmk4-1 hypocotyl elongation was partially rescued when tmk1-1 tmk4-1 seedlings were grown on medium with a lower $\mathrm{pH}(\mathrm{pH} 5.0$ and $\mathrm{pH} 4.3$ ) compared with standard medium (pH 5.7) (Extended Data Fig. 4a, b). Moreover, ost2-2D, which caused activation of the $\mathrm{PM} \mathrm{H}^{+}$-ATPase, partially rescued the hypocotyl elongation defect of tmk1-1 tmk4-1 mutants (Extended Data Fig. 4c, d). TMK1 and TMK4 probably activate other downstream pathways to regulate hypocotyl elongation in addition to the $\mathrm{PM} \mathrm{H}^{+}$-ATPase activation, such as ROP GTPase signalling to the organization of the cytoskeleton ${ }^{20,35}$. Such additional downstream pathways may explain the incomplete rescue of the hypocotyl elongation defect in tmk1-1 tmk4-1 plants by ost2-2D. Together, our results indicate that TMK1 and TMK4 are required for apoplastic acidification through auxin-triggered activation of $\mathrm{PM} \mathrm{H}^{+}$-ATPase, contributing to auxin regulation of hypocotyl cell elongation.

In this Article, we show that TMK1 directly interacts with PM $\mathrm{H}^{+}$-ATPases on the $\mathrm{PM}$, and this interaction was induced rapidly (within $10 \mathrm{~s}$ ) by auxin treatment (Fig. 1e,f), well preceding an auxin-induced increase in cell elongation ${ }^{16}$. Thus, the auxin-induced TMK-AHA association can be considered to be the very early response for auxin signal transduction. Our results suggest that, once interacting with AHAs after auxin stimulation, TMK1 directly phosphorylates AHA1 at the penultimate Thr residue (Fig. 2 and Extended Data Fig. 2). An accompanying paper by Li et al. shows that this auxin-induced phosphorylation of AHA's penultimate Thr residue occurred in root tissues within $2 \mathrm{~min}$ after auxin treatment ${ }^{36}$, nearly as rapid as the auxin-induced interaction between TMK1 and AHA1 (Fig. 1b, f). TMKs therefore regulate AHA
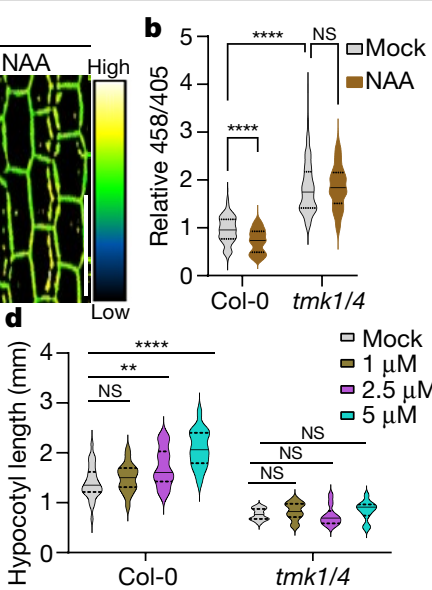

the hypocotyl segments at $30 \mathrm{~min}$ to that at $0 \mathrm{~min}$ is represented on the $y$ axis. The results were analysed using two-way ANOVA. $n=20$ hypocotyl sections per line.d, Auxin-induced hypocotyl elongation in seedlings. $n=16(\mathrm{Col}-0)$ and $n=10$ (tmk1-1 tmk4-1) (three independent assays). Statistical analysis was performed by two-way ANOVA (b-d). ${ }^{* *} P \leq 0.01,{ }^{* * * *} P \leq 0.0001$; NS, not significant. For a, scale bars, $100 \mu \mathrm{m}$.

activation by affecting the phosphorylation status of the penultimate Thr residue. As a consequence, auxin induced apoplastic acidification in a TMK1/TMK4-dependent manner in hypocotyl cells (Figs. 3a, b and $4 \mathrm{a}, \mathrm{b})$. Moreover, reducing the apoplast $\mathrm{pH}$ either genetically by ost $2-2 \mathrm{D}$ or growing seedlings in an acidic environment partially restored the hypocotyl elongation defect of tmk1-1 tmk4-1 plants (Extended Data Fig. 4a-d). These data suggest that, after activation by auxin, the cell-surface auxin-signalling components TMKs act as protein kinases to directly and rapidly initiate the phosphorylation and activation of $\mathrm{PM} \mathrm{H}^{+}$-ATPase, although our findings do not exclude the possibility that the TMK-mediated AHA phosphorylation may also respond to other stimuli under certain conditions. By contrast, the nuclear auxin signalling inhibits ATPase dephosphorylation through the TIR1/ AFB-SAUR-PP2C.D pathway ${ }^{17,37,38}$. Thus, the current findings support the hypothesis that the cell-surface and intracellular auxin-signalling pathways, respectively, initiate and sustain $\mathrm{PM} \mathrm{H}^{+}$-ATPase activation in cells in which auxin promotes cell expansion, such as in hypocotyls, and collectively explain the acid growth theory. In roots, TMK-dependent auxin signalling also promotes ATPase activation, but to counter the rapid alkalization (or membrane depolarization) activated by TIR1/ $\mathrm{AFBs}^{36,39,40}$. Importantly, these findings, together with the recent findings on the TMK-mediated noncanonical auxin signalling in regulating pavement cell morphogenesis ${ }^{18,41}$, differential growth of the apical hook $^{18}$, lateral root formation ${ }^{19}$, root gravitropic response ${ }^{42}$ and thermomorphogenesis ${ }^{23}$, are emerging as a common theme that auxin regulates growth and developmental processes through the coordinate actions of intracellular and cell-surface auxin-signalling systems.

\section{Online content}

Any methods, additional references, Nature Research reporting summaries, source data, extended data, supplementary information, acknowledgements, peer review information; details of author contributions and competing interests; and statements of data and code availability are available at https://doi.org/10.1038/s41586-021-03976-4.

1. Du, M., Spalding, E. P. \& Gray, W. M. Rapid auxin-mediated cell expansion. Annu. Rev. Plant Biol. 71, 379-402 (2020).

2. Haruta, M., Gray, W. M. \& Sussman, M. R. Regulation of the plasma membrane proton pump (H+-ATPase) by phosphorylation. Curr. Opin. Plant Biol. 28, 68-75 (2015).

3. McQueen-Mason, S., Durachko, D. M. \& Cosgrove, D. J. Two endogenous proteins that induce cell wall extension in plants. Plant Cell 4, 1425-1433 (1992). 
4. Barbez, E., Dunser, K., Gaidora, A., Lendl, T. \& Busch, W. Auxin steers root cell expansion via apoplastic $\mathrm{pH}$ regulation in Arabidopsis thaliana. Proc. Natl Acad. Sci. USA 114 E4884-E4893 (2017)

5. Rayle, D. L. \& Cleland, R. Enhancement of wall loosening and elongation by acid solutions. Plant Physiol. 46, 250-253 (1970).

6. Moloney, M. M., Elliott, M. C. \& Cleland, R. E. Acid growth effects in maize roots: evidence for a link between auxin-economy and proton extrusion in the control of root growth. Planta 152, 285-291 (1981).

7. Hager, A. Role of the plasma membrane $\mathrm{H}^{+}$-ATPase in auxin-induced elongation growth: historical and new aspects. J. Plant Res. 116, 483-505 (2003).

8. Haruta, M. et al. Molecular characterization of mutant Arabidopsis plants with reduced plasma membrane proton pump activity. J. Biol. Chem. 285, 17918-17929 (2010).

9. Palmgren, M. G., Sommarin, M., Serrano, R. \& Larsson, C. Identification of an autoinhibitory domain in the $\mathrm{C}$-terminal region of the plant plasma membrane $\mathrm{H}^{+}$-ATPase. J. Biol. Chem. 266, 20470-20475 (1991).

10. Ekberg, K., Palmgren, M. G., Veierskov, B. \& Buch-Pedersen, M. J. A novel mechanism of P-type ATPase autoinhibition involving both termini of the protein. J. Biol. Chem. 285, 7344-7350 (2010)

11. Jahn, T. et al. The 14-3-3 protein interacts directly with the $\mathrm{C}$-terminal region of the plant plasma membrane $\mathrm{H}^{+}$-ATPase. Plant Cell 9, 1805-1814 (1997).

12. Olsson, A., Svennelid, F., Ek, B., Sommarin, M. \& Larsson, C. A phosphothreonine residue at the $\mathrm{C}$-terminal end of the plasma membrane $\mathrm{H}^{+}$-ATPase is protected by fusicoccin-induced 14-3-3 binding. Plant Physiol. 118, 551-555 (1998).

13. Fuglsang, A. T. et al. Binding of 14-3-3 protein to the plasma membrane $\mathrm{H}^{+}$-ATPase AHA2 involves the three C-terminal residues Tyr(946)-Thr-Val and requires phosphorylation of Thr(947). J. Biol. Chem. 274, 36774-36780 (1999).

14. Svennelid, F. et al. Phosphorylation of Thr- 948 at the $C$ terminus of the plasma membrane $\mathrm{H}^{+}$-ATPase creates a binding site for the regulatory 14-3-3 protein. Plant Cell 11, 2379-2391 (1999).

15. Maudoux, O. et al. A plant plasma membrane $\mathrm{H}^{+}$-ATPase expressed in yeast is activated by phosphorylation at its penultimate residue and binding of 14-3-3 regulatory proteins in the absence of fusicoccin. J. Biol. Chem. 275, 17762-17770 (2000).

16. Fendrych, M., Leung, J. \& Friml, J. TIR1/AFB-Aux/IAA auxin perception mediates rapid cell wall acidification and growth of Arabidopsis hypocotyls. eLife 5, https://doi.org/10.7554/ eLife.19048 (2016).

17. Spartz, A. K. et al. SAUR inhibition of PP2C-D phosphatases activates plasma membrane $\mathrm{H}^{+}$-ATPases to promote cell expansion in Arabidopsis. Plant Cell 26, 2129-2142 (2014).

18. Cao, M. et al. TMK1-mediated auxin signalling regulates differential growth of the apical hook. Nature 568, 240-243 (2019).

19. Huang, R. et al. Noncanonical auxin signaling regulates cell division pattern during lateral root development. Proc. Natl Acad. Sci. USA 116, 21285-21290 (2019).

20. Xu, T. et al. Cell surface ABP1-TMK auxin-sensing complex activates ROP GTPase signaling. Science 343, 1025-1028 (2014).

21. Baral, A. et al. External mechanical cues reveal a katanin-independent mechanism behind auxin-mediated tissue bending in plants. Dev. Cell 56, 67-80 (2021).

22. Wang, Q. et al. A phosphorylation-based switch controls TAA1-mediated auxin biosynthesis in plants. Nat. Commun. 11, 679 (2020).

23. Gaillochet, C. et al. HY5 and phytochrome activity modulate shoot-to-root coordination during thermomorphogenesis in Arabidopsis. Development 147, https://doi.org/10.1242/ dev.192625 (2020)

24. $\mathrm{Xu}$, T. et al. Cell surface- and rho GTPase-based auxin signaling controls cellular interdigitation in Arabidopsis. Cell 143, 99-110 (2010).

25. Leyser, O. Auxin signaling. Plant Physiol. 176, 465-479 (2018).

26. Mockaitis, K. \& Estelle, M. Auxin receptors and plant development: A new signaling paradigm. Annu. Rev. Cell Dev. Bio. 24, 55-80 (2008).
27. Takahashi, K., Hayashi, K. \& Kinoshita, T. Auxin activates the plasma membrane H+-ATPase by phosphorylation during hypocotyl elongation in Arabidopsis. Plant Physiol. 159 632-641 (2012).

28. Dai, N., Wang, W., Patterson, S. E. \& Bleecker, A. B. The TMK subfamily of receptor-like kinases in Arabidopsis display an essential role in growth and a reduced sensitivity to auxin. PLoS ONE 8, e60990 (2013).

29. Falhof, J., Pedersen, J. T., Fuglsang, A. T. \& Palmgren, M. Plasma membrane $\mathrm{H}^{+}$-ATPase regulation in the center of plant physiology. Mol. Plant 9, 323-337 (2016).

30. Kinoshita, T. \& Shimazaki, K. Blue light activates the plasma membrane $\mathrm{H}^{+}$-ATPase by phosphorylation of the C-terminus in stomatal guard cells. EMBO J. 18, 5548-5558 (1999).

31. Kerkeb, L., Venema, K., Donaire, J. P. \& Rodriguez-Rosales, M. P. Enhanced H $\mathrm{H}^{+} /$ATP coupling ratio of H+-ATPase and increased 14-3-3 protein content in plasma membrane of tomato cells upon osmotic shock. Physiol. Plant 116, 37-41 (2002).

32. Niittyla, T., Fuglsang, A. T., Palmgren, M. G., Frommer, W. B. \& Schulze, W. X. Temporal analysis of sucrose-induced phosphorylation changes in plasma membrane proteins of Arabidopsis. Mol. Cell. Proteomics 6, 1711-1726 (2007).

33. Han, J. \& Burgess, K. Fluorescent indicators for intracellular pH. Chem. Rev. 110 2709-2728 (2010).

34. Merlot, S. et al. Constitutive activation of a plasma membrane $\mathrm{H}^{+}$-ATPase prevents abscisic acid-mediated stomatal closure. EMBO J. 26, 3216-3226 (2007).

35. Fu, Y., Xu, T., Zhu, L., Wen, M. \& Yang, Z. A ROP GTPase signaling pathway controls cortical microtubule ordering and cell expansion in Arabidopsis. Curr. Biol. 19, 1827-1832 (2009).

36. Li, L. et al. Cell surface and intracellular auxin signalling for $\mathrm{H}+$ fluxes in root growth. https://doi.org/10.1038/s41586-021-04037-6 Nature (2021).

37. Ren, H., Park, M. Y., Spartz, A. K., Wong, J. H. \& Gray, W. M. A subset of plasma membrane-localized PP2C.D phosphatases negatively regulate SAUR-mediated cell expansion in Arabidopsis. PLoS Genet. 14, e1007455 (2018).

38. Spartz, A. K. et al. The SAUR19 subfamily of SMALL AUXIN UP RNA genes promote cell expansion. Plant J. 70, 978-990 (2012).

39. Fendrych, M. et al. Rapid and reversible root growth inhibition by TIR1 auxin signalling. Nat. Plants 4, 453-459 (2018).

40. Prigge, M. J. et al. Genetic analysis of the Arabidopsis TIR1/AFB auxin receptors reveals both overlapping and specialized functions. eLife $\mathbf{9}$, https://doi.org/10.7554/eLife.54740 (2020).

41. Chen, J. \& Yang, Z. Novel ABP1-TMK auxin sensing system controls ROP GTPase-mediated interdigitated cell expansion in Arabidopsis. Small GTPases 5, https://doi.org/10.4161/ sgtp.29711 (2014).

42. Marques-Bueno, M. M. et al. Auxin-regulated reversible inhibition of TMK1 signaling by MAKR2 modulates the dynamics of root gravitropism. Curr. Biol. 31, 228-237 (2021).

Publisher's note Springer Nature remains neutral with regard to jurisdictional claims in published maps and institutional affiliations.

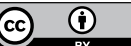

Open Access This article is licensed under a Creative Commons Attribution 4.0 International License, which permits use, sharing, adaptation, distribution and reproduction in any medium or format, as long as you give appropriate credit to the original author(s) and the source, provide a link to the Creative Commons license, and indicate if changes were made. The images or other third party material in this article are included in the article's Creative Commons license, unless indicated otherwise in a credit line to the material. If material is not included in the article's Creative Commons license and your intended use is not permitted by statutory regulation or exceeds the permitted use, you will need to obtain permission directly from the copyright holder. To view a copy of this license, visit http://creativecommons.org/licenses/by/4.0/.

(c) The Author(s) 2021 


\section{Methods}

\section{Plant materials and growth conditions}

A. thaliana Columbia ecotype (Col-0) was used as the wild type in this study. ost2-2D seeds were obtained from J. Leung. The tmk1-1 tmk4-1 mutant in the Col-0 background and pTMK1-TMK1-GFP transgenic lines (in the tmk1-1 tmk4-1 mutant) were described previously ${ }^{18,19}$. The ost2-2D tmk1-1 tmk4-1 mutants were generated by genetic crosses and confirmed by genotyping. Arabidopsis plants were grown in soil (SungroS16-281) in a growth room at $23^{\circ} \mathrm{C}, 40 \%$ relative humidity and $75 \mu \mathrm{E} \mathrm{m}^{-2} \mathrm{~s}^{-1}$ light under a $12 \mathrm{~h}$ photoperiod for approximate 4 weeks before protoplast isolations. To grow Arabidopsis seedlings, the seeds were surface-sterilized with $50 \%$ bleach for $10 \mathrm{~min}$ ( $\mathrm{tmk} 1-1 \mathrm{tm} k 4-1$ seeds were sterilized with $75 \%(\mathrm{v} / \mathrm{v})$ ethanol for $5 \mathrm{~min}$ ), and washed three times with sterilized distilled $\mathrm{H}_{2} \mathrm{O}$, and then placed onto plates with $1 / 2 \mathrm{MS}$ medium containing $0.5 \%$ sucrose and $0.8 \%$ agar at $\mathrm{pH} 5.7$ in the dark with vertical growth. Then $2-3 \mathrm{~d}$ after germination, hypocotyls were used for cell characterization.

\section{Plasmid construction and generation of transgenic plants}

Full-length and truncated variants $T M K 1, A H A 1$ and $A H A 2$ were amplified by PCR from Col-0 cDNA and cloned into a protoplast transient expression vector (HBT vectors obtained from $L$. Shan and P. He) or the plant binary vectors pGWB641 and pGWB644. Stable transgenic lines were generated using standard Agrobacterium tumefaciens-mediated transformation in the tmk1-1 tmk4-1 mutant or Col-0 (ref. ${ }^{43}$ ). The full-length cDNAs of $T M K 1$ and $A H A 1$ were amplified by PCR, and then cloned into the pDONR221-P1P4 and pDONR221-P3P2 vectors using the BP recombination reaction (Invitrogen), respectively. pDONR221-P1P4-TMK1 was recombined with $p D O N R 221-\mathrm{P} 3 \mathrm{P} 2-\mathrm{AHA1}$ into $\mathrm{pFRETgc-2in1-NC}$ to generate pFRET-mEGFP-AHA1+TMK1-mCherry ${ }^{44}$.pDONR221-P3P2-AHA1 was recombined with pENTRL1-pLac-LacZalpha-L4 (Invitrogen) intopFRETgc-2in1-NC togeneratepFRET-mEGFP-AHA1.pDONR221-P1P4-TMK1was recombined with pENTRL3-pLac-Tet-L2 (Invitrogen) into pFRETgc-2in1-CC togenerate pFRET-MK1-mCherry. The $A H A 2 \mathrm{C}$-terminal region was cloned into pDest565, and expressed in Escherichia coli (Rosetta, BL21) (a list of the primers is provided in Supplementary Table 1).

\section{Determination of $\mathrm{H}^{+}$-ATPase phosphorylation levels}

The immunoblot analysis was performed as described by Hayashi ${ }^{45}$ using specific antibodies against the catalytic domain of AHA2 and phosphorylated Thr947 in AHA2 (1:5,000 dilution) ${ }^{45}$. These antibodies recognize not only AHA2 but also other $\mathrm{H}^{+}$-ATPase isoforms in Arabidopsis $^{46}$. In brief, the roots were removed from $1 / 2 \mathrm{MS}$-grown seedlings (aged $5 \mathrm{~d}$ ), and the remaining aerial sections were incubated in a KPSC buffer (10 mM potassium phosphate, $\mathrm{pH} 6.0,2 \%$ sucrose, $50 \mu \mathrm{m}$ chloramphenicol) in the dark for $10 \mathrm{~h}$. The buffer was then replaced every hour. The pretreated tissues were incubated in the presence of $100 \mathrm{nM}$ IAA for $10 \mathrm{~min}$ or $10 \mu \mathrm{M}$ IAA for $30 \mathrm{~min}$ in the dark. The aerial sections were collected and grounded with a plastic pestle, followed by solubilization in $40 \mu$ l of SDS buffer ( $3 \%(\mathrm{w} / \mathrm{v})$ SDS, $30 \mathrm{mM}$ Tris- $\mathrm{HCl}(\mathrm{pH} 8.0)$, $10 \mathrm{mM}$ EDTA, $10 \mathrm{mM} \mathrm{NaF}, 30 \%$ (w/v) sucrose, 0.012\% (w/v) Coomassie Brilliant Blue and 15\% (v/v) 2-mercaptoethanol), and the homogenates were centrifuged at room temperature $(10,000 \mathrm{~g}$ for $5 \mathrm{~min})$. Next, $12 \mu \mathrm{l}$ of the supernatant was loaded onto $10 \%(\mathrm{w} / \mathrm{v})$ SDS-PAGE gels to assess the $\mathrm{H}^{+}$-ATPase or the phosphorylated penultimate Thr levels using the respective above-mentioned antibodies. Goat anti-rabbit IgG $(1: 10,000$ dilution) conjugated to horseradish peroxidase (Santa Cruz, sc-2357) was used as a secondary antibody. The chemiluminescent signal was quantified using ImageJ (Fiji, Java 1.8.0_172) (Fig. 2a, Extended Data Fig. $2 \mathrm{f}$ and Supplementary Fig. 1).

\section{HPTS staining and imaging}

HPTS staining and imaging were performed as described by Barbez ${ }^{4}$ with modifications. In brief, two-day etiolated seedlings were transferred and incubated with $1 \mathrm{mM} \mathrm{HPTS}$ (from $100 \mathrm{mM}$ water stock) with $0.01 \%$ Triton X-100 under vacuum (10-15 pa) $5 \mathrm{~min}$. The seedlings were then incubated with HPTS for 60 min in the liquid growth medium. The seedlings were subsequently mounted in the same growth medium on a microscopy slide and covered with a coverslip. For auxin treatment, seedlings were incubated in $1 / 2 \mathrm{MS}$ growth medium supplemented with $1 \mathrm{mM}$ HPTS and NAA in the stated concentration for $15 \mathrm{~min}$ and subsequently mounted in the same growth medium on a microscopy slide and covered with a coverslip. Seedling imaging was performed using an inverted Zeiss 880 confocal microscope equipped with a highly sensitive GaAsP detector. Fluorescent signals for the protonated HPTS form (excitation, $405 \mathrm{~nm}$; emission peak, $514 \mathrm{~nm}$ ), as well as the deprotonated HPTS form (excitation, $458 \mathrm{~nm}$; emission peak, $514 \mathrm{~nm}$ ), were detected using a $\times 10$ water-immersion objective.

\section{IP-MS analyses}

The $p T M K 1:: g T M K 1-G F P /$ tmk1-1 tmk4-1 seedlings were grown on 1/2 MS medium for $10 \mathrm{~d}$, and the entire seedlings were collected and ground in liquid nitrogen with a mortar and pestle. Total proteins were extracted using extraction buffer $(50 \mathrm{mM}$ Tri- $\mathrm{HCl} \mathrm{pH} 7.4,150 \mathrm{mM} \mathrm{NaCl}, 5 \mathrm{mM}$ EDTA, 0.5\% Triton X-100 with protease inhibitor and phosphatase inhibitor) on ice. The extracts were centrifugated at $13,000 \mathrm{~g}$ for $30 \mathrm{~min}$, and the supernatants were incubated with GFP-Trap agarose beads (GFP-Trap_A, gta-20,ChromoTek) at $4{ }^{\circ} \mathrm{C}$ for $2 \mathrm{~h}$ to immunoprecipitate TMK1-GFP proteins. The agarose beads were washed and resuspended with $50 \mathrm{mM}$ Tris-Cl buffer ( $\mathrm{pH}$ 7.8). One tenth of the beads was used for immunoblot analysis with anti-GFP antibodies. The remaining agarose beads were used for LC coupled with tandem MS (LC-MS/MS) analysis. MS analysis was carried out by Orbitrap Fusion mass spectrometry (Thermo Fisher Scientific).

\section{Phosphoproteomics analyses}

Col-0 and the tmk1-1 tmk4-1 seedlings were cultured on 1/2 MS plate for $5 \mathrm{~d}$, then the aerial parts of seedlings were transferred to $1 / 2 \mathrm{MS}$ liquid medium and incubated in the KPSC buffer $(10 \mathrm{mM}$ potassium phosphate, $\mathrm{pH} 6.0,2 \%$ sucrose and $50 \mu \mathrm{m}$ chloramphenicol) in the dark overnight, and the buffer was replaced every $1 \mathrm{~h}$ for $12 \mathrm{~h}$ (ref. ${ }^{47}$ ). Seedings were collected and flash-frozen in liquid nitrogen. A total of $1 \mathrm{~g}$ of frozen shoots (fresh weight) was ground with a liquid-nitrogen-precooled mortar and pestle, and then homogenized in $5 \mathrm{ml}$ extraction buffer (50 mM Tris- $\mathrm{HCl}$ buffer ( $\mathrm{pH} 8$ ), $0.1 \mathrm{M} \mathrm{KCl}, 30 \%$ sucrose, 5 mM EDTA and $1 \mathrm{mM}$ dithiothreitol (DTT) in Milli- $\mathrm{Q}$ water, $1 \times$ complete protease inhibitor mixture and the PhosSTOP phosphatase inhibitor mixture) in a Dounce Homogenizer. At least 50 strokes were performed. The homogenate was filtered through four layers of miracloth and centrifuged at $5,000 \mathrm{~g}$ at $4{ }^{\circ} \mathrm{C}$ for $10 \mathrm{~min}$. Half of the supernatant was used to resuspend the pellet, and the mixture was centrifuged again at $5,000 \mathrm{~g}$ $4{ }^{\circ} \mathrm{C}$ for $10 \mathrm{~min}$. The two fractions of the supernatants were combined and mixed with 3,1 and 4 volumes of methanol, chloroform and water, respectively. The mixtures were centrifuged at 5,000 $\mathrm{g}$ for $10 \mathrm{~min}$, and the aqueous phase was removed. After the addition of four volumes of methanol, the proteins were pelleted by centrifugation at $4,000 \mathrm{~g}$ for $10 \mathrm{~min}$. The pellets were washed with $80 \%$ acetone and resuspended in 6 M guanidinium hydrochloride in $50 \mathrm{mM}$ triethylammonium bicarbonate buffer ( $\mathrm{pH} 8$ ). The proteins were used for tandem mass tag labelling according to the Kit protocol (Thermo Fisher Scientific, 90096) and quantification by MS.

Nano-LC-MS/MS was performed using the Dionex rapid-separation liquid chromatography system interfaced with a QExactive HF (Thermo Fisher Scientific). Samples were loaded onto an Acclaim PepMap 100 trap column $(75 \mu \mathrm{m} \times 2 \mathrm{~cm}$, Thermo Fisher Scientific) and washed with buffer $\mathrm{A}(0.1 \%$ trifluoroacetic acid $)$ for $5 \mathrm{~min}$ with a flow rate of $5 \mu \mathrm{min}^{-1}$. The trap was brought in line with the nano analytical column (nanoEase, MZ peptide BEH C18, $130 \mathrm{~A}, 1.7 \mu \mathrm{m}, 75 \mu \mathrm{m} \times 20 \mathrm{~cm}$, Waters) with a flow rate of $300 \mathrm{nl} \mathrm{min}{ }^{-1}$ with a multistep gradient (4-15\% buffer 


\section{Article}

$\mathrm{B}(0.16 \%$ formic acid and $80 \%$ acetonitrile $)$ in $20 \mathrm{~min}$, then $15-25 \% \mathrm{~B}$ in $40 \mathrm{~min}$, followed by $25-50 \% \mathrm{~B}$ in $30 \mathrm{~min})$. MS data were acquired using a data-dependent acquisition procedure with a cyclic series of a full scan acquired with a resolution of 120,000 followed by MS/MS scans (33\% collision energy in the HCD cell) with a resolution of 45,000 of the 20 most intense ions with a dynamic exclusion duration of $20 \mathrm{~s}$.

All LC-MS data were analysed using Maxquant (v.1.6.2.6) with the Andromeda search engine. The type of LC-MS run was set to reporter ion MS2 with 10 plex tandem mass tags as isobaric labels. Reporter ion mass tolerance was set at $0.003 \mathrm{Da}$. LC-MS data were searched against TIAR10 with the addition of potential contaminants. Protease was set as trypsin/P, allowing two misscuts for total proteomic data and three misscuts for the phosphor-enriched sample (post-translational modification sample). Carbamidomethylation of cysteine was set as a fixed modification, $\mathrm{N}$-terminal acetylation, oxidation at methionine as well as phosphorylation at serine, threonine and tyrosine were set as variable modifications. Proteins with a false-discovery rate of $<1 \%$ were reported. For quantification, spectra were filtered by minimum reporter PIF set at 0.6 .

The results were further analysed using Perseus (v.1.6.1.3). The protein group results were first filtered for reverse and contaminant hits and the reporter ion intensity values were further $\log _{2}$-transformed and normalized to the column total. For group comparisons, statistical significance between groups was analysed using Student's $t$-tests with equal variance on both sides, requiring two valid values in total, and the $Q$ value was calculated using the permutation test. SO $\left(\text { ref. }^{48}\right)^{2}$ was set to 1.

The phospho(STY)-site reporter ions were first normalized to proteome column total, and then filtered for reverse and contaminant hits, and the reporter ion intensity values were further $\log _{2}$-transformed. Each phospho(STY) site was further normalized to protein abundance from proteome data if available. Only sites that have site localization confidence $>75 \%$ were included in the analysis. For group comparison, statistical significance between groups was analysed using Student's $t$-tests with equal variance on both sides, requiring two valid values in total, and the $Q$ value was calculated using the permutation test. SO was set to 1 .

\section{Co-IP assays with transgenic plants}

Approximately 1 g of $35 S:: G F P$ and $35 S:: G F P$-AHA1 plants (aged 4 weeks) was ground in liquid $\mathrm{N}_{2}$ and further ground in $0.5 \mathrm{ml}$ of ice-cold co-IP buffer (10 mM HEPES at $\mathrm{pH} 7.5,100 \mathrm{mM} \mathrm{NaCl}, 1 \mathrm{mMEDTA}, 10 \%$ glycerol, $0.1 \%$ Triton X-100 and protease inhibitor mixture from Roche). The homogenates were centrifuged at $12,470 \mathrm{~g}$ at $4{ }^{\circ} \mathrm{C}$ for $10 \mathrm{~min}$. The supernatant was incubated with anti-GFP-Trap antibodies (Chromotek, 3h9, 1:1,000 dilution) for $2 \mathrm{~h}$ with gentle shaking. The beads were collected and washed three times with washing buffer (10 mM HEPES ( $\mathrm{pH} 7.5)$, $100 \mathrm{mM} \mathrm{NaCl}, 1 \mathrm{mMEDTA}, 10 \%$ glycerol and $0.1 \%$ Triton X-100) and once with $50 \mathrm{mM}$ Tris $\cdot \mathrm{HCl}(\mathrm{pH} 7.5)$, and analysed by western blotting using anti-TMK1 or anti-TMK4 antibodies ${ }^{18}$. The total input proteins were determined using anti-GFP antibodies (Fig.1a, Extended Data Fig. 1b and Supplementary Fig. 1).

\section{Co-IP analysis of the TMK-AHA interaction induced by auxin treatment in Arabidopsis protoplasts}

Wild-type Col-0 leaf protoplasts were prepared as described by Yoo et al. ${ }^{49}$. Protoplasts $\left(2 \times 10^{5}\right)$ were co-transfected with $35 S:: A H A 1-H A$ or 35S::TMK1-Myc plasmid DNA, which was prepared using the Invitrogen PureLink Plasmid Maxiprep Kit, and incubated at room temperature for $10 \mathrm{~h}$. Transfected protoplasts were then collected in $2 \mathrm{ml}$ Eppendorf tubes and centrifuged at $100 \mathrm{~g}$ for $1 \mathrm{~min}$. The supernatant was discarded, and the protoplasts were resuspended with $100 \mu \mathrm{lW} 5$ solution $(2 \mathrm{mM}$ MES-KOH, pH 5.7, $5 \mathrm{mM} \mathrm{KCl}, 154 \mathrm{mM} \mathrm{NaCl}$ and $125 \mathrm{mM} \mathrm{CaCl}_{2}$ ). The protoplasts were treated with $1 \mu \mathrm{MNAA}$ for the indicated time periods, frozen in liquid $\mathrm{N}_{2}$ immediately and stored in $-80^{\circ} \mathrm{C}$. The samples were lysed with $0.5 \mathrm{ml}$ of extraction buffer (10 mM HEPES (pH 7.5), $100 \mathrm{mM} \mathrm{NaCl}$, 1 mMEDTA, $10 \%(\mathrm{v} / \mathrm{v})$ glycerol, $0.5 \%$ Triton X-100 and protease inhibitor mixture from Roche). After vortexing vigorously for $30 \mathrm{~s}$, the samples were centrifuged at $12,470 \mathrm{~g}$ for $10 \mathrm{~min}$ at $4{ }^{\circ} \mathrm{C}$. The supernatant was incubated with anti-Myc antibodies (Sinobiological, 100029-MM08, 1:1,000 dilution) for $2 \mathrm{~h}$, and then incubated with protein $\mathrm{G}$ agarose beads (Pierce, 20397) for another $2 \mathrm{~h}$ at $4{ }^{\circ} \mathrm{C}$ with gentle shaking. Beads were collected by centrifugation at $100 \mathrm{~g}$ for $1 \mathrm{~min}$ at $4{ }^{\circ} \mathrm{C}$ followed by washing twice with extraction buffer. The beads were washed with $50 \mathrm{mM}$ Tris-Cl buffer ( $\mathrm{pH} 7.5$ ), and the immunoprecipitated proteins were analysed by immunoblotting with anti-HA-HRP antibodies (Invitrogen, 26183, 1:2,000 dilution) (Fig.1b and Supplementary Fig. 1).

\section{In vitro pull-down assay}

MBP- or GST-fusion proteins were expressed in E. coli and affinity-purified using standard protocols. In brief, $200 \mathrm{ml}$ of isopropyl- $\beta$-D-thiogalactoside-induced cell culture pellet was lysed in 20 ml lysis buffer (containing $0.5 \%$ Triton X-100) by sonication on ice. Centrifuge lysates were cleared by centrifuging at $10,000 \mathrm{~g}$ for $30 \mathrm{~min}$ at $4{ }^{\circ} \mathrm{C}$. The supernatant was then incubated with $100 \mu$ l amylose resins or glutathione-Sepharose beads at $4{ }^{\circ} \mathrm{C}$ for $4 \mathrm{~h}$ with gentle rotation. The beads were then centrifuged and washed three times with lysis buffer. Proteins were eluted with GST $(10 \mathrm{mM}$ reduced glutathione in $50 \mathrm{mM}$ Tris pH 8.0) or MBP (20 mM Tris-HCl, $200 \mathrm{mM} \mathrm{NaCl}, 1 \mathrm{mMEDTA}$, $1 \mathrm{mM}$ DTT, $10 \mathrm{mM}$ maltose, $\mathrm{pH}$ 7.4) buffer. The protein concentration was estimated using the NanoDrop ND-1000 spectrophotometer and confirmed using the Bio-Rad Quick Start Bradford Dye Reagent. GST- or GST-fusion proteins $(10 \mu \mathrm{g}$; immobilized on glutathione-Sepharose beads) were incubated with $10 \mu \mathrm{g}$ prewashed MBP or MBP fusion proteins at $4{ }^{\circ} \mathrm{C}$ in $150 \mu$ of incubation buffer (10 mM HEPES ( $\left.\mathrm{pH} 7.5\right)$, $100 \mathrm{mM} \mathrm{NaCl}, 1 \mathrm{mMEDTA}, 10 \%$ glycerol and $0.5 \%$ Triton X-100) for $1 \mathrm{~h}$. The beads were collected and washed three times with washing buffer (20 mM HEPES (pH 7.5), 300 mM NaCl,1 mMEDTA and 0.5\% NP-40) and once with $50 \mathrm{mM}$ Tris- $\mathrm{HCl}(\mathrm{pH}$ 7.5). Proteins in the beads were analysed by immunoblotting with anti-GST (Santa Cruz, sc-138, 1:1,000 dilution) or anti-MBP (Invitrogen, PA1-989, 1:1,000 dilution) antibodies (Extended Data Fig. 1d and Supplementary Fig. 1).

\section{Vanadate-sensitive ATPase activity measurement}

ATP hydrolysis by $\mathrm{PM} \mathrm{H}^{+}$-ATPase was measured in a vanadate-sensitive manner as previously described ${ }^{27}$. In brief, the aerial parts of seedlings (Col-0, tmk1-1, tmk4-1, and tmk1-1 tmk4-1; aged $14 \mathrm{~d}$ ) were incubated in KPSC buffer (10 mM potassium phosphate, $\mathrm{pH} 6.0,2 \%$ sucrose, $50 \mu \mathrm{m}$ chloramphenicol) in the dark for $10 \mathrm{~h}$. The buffer was replaced every hour. The pretreated tissues were incubated in the presence of $10 \mu$ M IAA for $30 \mathrm{~min}$ in darkness. The tissues were homogenized with homogenization buffer (50 mM MOPS- $\mathrm{KOH}, \mathrm{pH} 7.0,100 \mathrm{mM} \mathrm{KNO}_{3}$, 2 mM sodium molybdate, $0.1 \mathrm{mM} \mathrm{NaF}, 2 \mathrm{mMEGTA}, 1 \mathrm{mM}$ phenylmethylsulfonyl fluoride and $20 \mu \mathrm{M}$ leupeptin) and the homogenates were centrifuged at $10,000 \mathrm{~g}$ for $10 \mathrm{~min}$; the obtained supernatant was further ultracentrifuged at $45,000 \mathrm{~g}$ for $60 \mathrm{~min}$. The resultant pellet (the microsomal fraction) was resuspended in the homogenization buffer. The ATP hydrolytic activity of the microsomal fraction was measured in a vanadate-sensitive manner, and the inorganic phosphate released from ATP was measured ${ }^{27}$.

\section{In vitro phosphorylation}

Protoplasts were isolated from plants expressing AHA1-GFP as described above. Agarose-immobilized (GFP-Trap beads, Chromotek, gta-100) AHA1-GFP proteins were incubated with $1 \mu \mathrm{g} \mathrm{MBP-TMK1}{ }^{\mathrm{KD}}$ or MBP-TMK1 ${ }^{\mathrm{KDKm}}$ recombinant proteins (expressed in $E$. coli and isolated by affinity purification) in phosphorylation buffer ( 5 mM HEPES, $10 \mathrm{mM} \mathrm{MgCl}_{2}, 10 \mathrm{mM} \mathrm{MnCl}_{2}, 1 \mathrm{mMDTT}$ and $\left.50 \mu \mathrm{M} \mathrm{ATP}\right)^{18}$ at room temperature $\left(24^{\circ} \mathrm{C}\right)$ for $1 \mathrm{~h}$. After incubation, the reaction was stopped by adding $4 \times$ SDS loading buffer. Proteins in the beads were analysed by 
immunoblotting using anti-pT947, anti-AHA1-cat or anti-GFP (Chromotek, 3h9) antibodies (Fig. 2c and Supplementary Fig. 1).

For in vitro phosphorylation of synthetic peptides, $10 \mathrm{mg}$ synthetic peptide AHA1-C16 (KLKGLDIDTAGHHYTV) or scrambled peptide (GDAHVKITHLDKGLIT) was incubated with $1 \mu \mathrm{g} \mathrm{MBP-TMK1} 1^{\mathrm{KD}}$ or MBP-TMK1 ${ }^{\mathrm{K}}$ ${ }^{\mathrm{DKm}}$ recombinant proteins in phosphorylation buffer for $1 \mathrm{~h}$. The peptide mixtures were then analysed using MS.

\section{Auxin-induced rapid hypocotyl segment elongation}

For analysing auxin-induced elongation of hypocotyl segments, the auxin-depleted hypocotyl sections $(2 \mathrm{~mm})$ were transferred to growth medium (10 mM KCl, 1 mM MES-KOH, pH 5.7, 0.8\% agar) with/without $10 \mu \mathrm{M}$ NAA for $30 \mathrm{~min}$. The hypocotyl sections were photographed and measured using Image $J$ at 0 min and 30 min after treatments. For analysing auxin-induced hypocotyl elongation in seedlings, $1 / 2 \mathrm{MS}$-grown seedlings (aged $4 \mathrm{~d}$ ) were transferred into $1 / 2 \mathrm{MS}$ medium containing the indicated concentrations of NAA and incubated for additional $48 \mathrm{~h}$ under normal growth conditions. Hypocotyl lengths were measured using Image J after treatment.

\section{Fabrication of the protoplast-capture microfluidics chip}

The design parameters for the protoplast capture chip (Extended Data Fig. 1e) are as follows: each capture unit consists of three pillars. The average diameter of each pillar is $50 \mu \mathrm{m}$. The distance between the two entrance pillars is $52 \mu \mathrm{m}$. The shortest distance between the entrance pillar and the bottom pillar is $27 \mu \mathrm{m}$. The height of the capture chamber is $80 \mu \mathrm{m}$. Each chip contains an array of 1,866 capture units. A master mould was fabricated on a silicon wafer using the traditional photolithography technique by Beijing Borui Yisheng Technology. In brief, SU-83050 was centrifuged at 1,600 r.p.m., soft-baked at $95^{\circ} \mathrm{C}$ for $30 \mathrm{~min}$ and exposed at $260 \mathrm{~mJ} \mathrm{~cm}^{-2}$. After exposure, the wafer was post-exposure baked at $95^{\circ} \mathrm{C}$ for $5 \mathrm{~min}$, developed for $3 \mathrm{~min}$ and air-dried with pressurized nitrogen.

The protoplast capture chip was fabricated with soft lithography using polydimethylsiloxane (PDMS) (Sylgard 184 Silicone Elastomer Kit, The DOW Chemical Company). In brief, PDMS prepolymer and curing agent were mixed at a 10:1 ratio, degassed in a vacuum chamber with negative pressure, poured onto the master mould and baked at $80^{\circ} \mathrm{C}$ for $2 \mathrm{~h}$. After curing, the PDMS slab was peeled off, hole-punched and, finally, plasma-oxidized to adhere to the cover glass.

\section{Auxin treatment and FRET analysis of protoplasts captured in microfluidics chips}

Arabidopsis (Col-0) protoplasts were isolated and transfected with 2in1 AHA1-GFP, TMK1-mCherry and AHA1-GFP/TMK1-mCherry vectors as described. Before the experiment, the protoplast capture chip was filled with a protoplast-suspending WI solution ( $4 \mathrm{mM} \mathrm{pH} 5.7 \mathrm{MES}-\mathrm{KOH}, 0.5 \mathrm{M}$ mannitol and $20 \mathrm{mMKCl}$ ). After air bubbles were entirely removed from the chip, the protoplast suspension was injected slowly into the chip from inlet 1 (Fig. 1e). The time-lapse FRET images were acquired at $5 \mathrm{~s}$ per frame using the Zeiss LSM880 confocal laser scanning microscope (argon $48830 \%$, and argon 5613\%). NAA or mock solution was injected into the chip from inlet 2 (Fig. 1e) at $25 \mathrm{~s}$ after the live imaging started.

The FRET efficiency was analysed using FRET sensitized emission methods ${ }^{46}$. In brief, the AHA1-GFP only, TMK1-mCherry only sample and the FRET samples were imaged using the same microscope settings (the donor and FRET channels were excited with $30 \%$ argon $488 \mathrm{~nm}$, and the emissions were collected using $498-551 \mathrm{~nm}$ and $600-670 \mathrm{~nm}$, respectively; the acceptor channel was excited with $3 \%$ argon $561 \mathrm{~nm}$ and the emissions were collected using 600-670 nm). To avoid interference by chlorophyll autofluorescence, protoplasts with concentrated chloroplasts at one side of the cell were processed for quantification. A segmented line was drawn along the PM region opposite to the site of chloroplasts to measure the mean signal intensity for each channel using Image-Pro Plus (http://www.mediacy.com/imageproplus) and LAS-X (Leica). The correction factors $\beta, \alpha, \gamma$ and $\delta$ were calculated with the donor- and acceptor-only reference samples, then the FRET efficiency was calculated using the equation below ${ }^{50}$.

$$
E_{(\mathrm{FRET}-\mathrm{SE})}=\frac{\text { FRET }- \text { donor } \times \beta-\text { acceptor } \times(\gamma-\alpha \times \beta)}{\text { acceptor } \times(1-\beta \times \delta)}
$$

The mean FRET efficiency and s.d. from 10 cells of 100 nM NAA or mock treatment are presented in Fig. 1g. To generate the FRET efficiency heat-map image, the plasma membrane region in the side opposite to the chloroplasts was cropped as the region of interest to avoid autofluorescence (Extended Data Fig. 1d). The cropped images from the donor, FRET and acceptor channels were processed using the image calculator module of ImageJ with the $E_{\text {(FRET-SE) }}$ equation shown above.

\section{Reporting summary}

Further information on research design is available in the Nature Research Reporting Summary linked to this paper.

\section{Data availability}

Data supporting the findings of this study are available within the paper and its Supplementary Information. Mass spectrometry raw data are available at the MassIVE under accession number msv000087822. Source data are provided with this paper.

43. Clough, S. J. \& Bent, A. F. Floral dip: a simplified method for Agrobacterium-mediated transformation of Arabidopsis thaliana. Plant J. 16, 735-743 (1998).

44. Hecker, A. et al. Binary 2in1 vectors improve in planta (co)localization and dynamic protein interaction studies. Plant Physiol. 168, 776-787 (2015).

45. Hayashi, M., Inoue, S., Takahashi, K. \& Kinoshita, T. Immunohistochemical detection of blue light-induced phosphorylation of the plasma membrane $\mathrm{H}^{+}$-ATPase in stomatal guard cells. Plant Cell Physiol. 52, 1238-1248 (2011).

46. Hayashi, Y. et al. Biochemical characterization of in vitro phosphorylation and dephosphorylation of the plasma membrane $\mathrm{H}^{+}$-ATPase. Plant Cell Physiol. 51, 1186-1196 (2010).

47. Thakur, J. K., Tyagi, A. K. \& Khurana, J. P. OsIAA1, an Aux/IAA cDNA from rice, and changes in its expression as influenced by auxin and light. DNA Res. 8, 193-203 (2001).

48. Tusher, V. G., Tibshirani, R. \& Chu, G. Significance analysis of microarrays applied to the ionizing radiation response. Proc Natl. Acad. Sci. USA 98, 5116-5121 (2001).

49. Yoo, S. D., Cho, Y. H. \& Sheen, J. Arabidopsis mesophyll protoplasts: a versatile cell system for transient gene expression analysis. Nature Protoc. 2, 1565-1572 (2007).

50. van Rheenen, J., Langeslag, M. \& Jalink, K. Correcting confocal acquisition to optimize imaging of fluorescence resonance energy transfer by sensitized emission. Biophys. J. 86, 2517-2529 (2004).

Acknowledgemacents We thank the members of the Yang laboratory for discussion and comments on this work; J. Leung (Department of Institute Jean-Pierre Bourgin, INRA) for ost2$2 D$ seeds; K. Iba (Kyushu University) for 35S::GFP-AHA1 seeds; C. Grefen (University of Tubingen) for FRET analyses 2in1 binary vectors; and L. Shan (University of Texas A\&M) for protoplast transient expression vectors. This work was in part supported by an NIH grant (GM100130) to Z.Y.; K.T. (2OKO6685) and T.K. (2OH05687 and 2OHO5910) were funded by MEXT/ JSPS KAKENHI. W.M.G. was funded by NIH (GM067203). H.Z. was funded by NIH (S10OD016400).

Author contributions W.L. and Z.Y. conceived the project and designed the experiments. W.L. conducted most of the experiments, with contributions from X.Z., W.T., K.T., X.P., J.D., H.R. and W.M.G.; W.L., S.P. and H.Z. conducted and analysed all the MS analyses of this paper; X.Z., J.D., X.P. and X.Y.Z. designed and conducted the FRET analyses. K.T. and T.K. conducted and analysed the ATPase activities. W.L., Z.Y. and W.M.G. wrote the manuscript with input from all of the other authors.

\section{Competing interests The authors declare no competing interests.}

\section{Additional information}

Supplementary information The online version contains supplementary material available at https://doi.org/10.1038/s41586-021-03976-4.

Correspondence and requests for materials should be addressed to Zhenbiao Yang. Peer review information Nature thanks Malcolm Bennett and the other, anonymous, reviewer(s) for their contribution to the peer review of this work. Peer reviewer reports are available.

Reprints and permissions information is available at http://www.nature.com/reprints. 


\begin{tabular}{|c|c|c|}
\hline Identified Proteins & Accession & Unique peptide \\
\hline $\mathrm{H}(+)$-ATPase 1 AHA1 & AT2G18960 & 14 \\
\hline $\mathrm{H}(+)$-ATPase 2 AHA2 & AT4G30190 & 19 \\
\hline $\mathrm{H}(+)$-ATPase 3 AHA3 & AT5G57350 & 3 \\
\hline $\mathrm{H}(+)$-ATPase 7 AHA7 & AT3G60330 & 1 \\
\hline $\mathrm{H}(+)$-ATPase 11 AHA11 & AT5G62670 & 18 \\
\hline
\end{tabular}

C

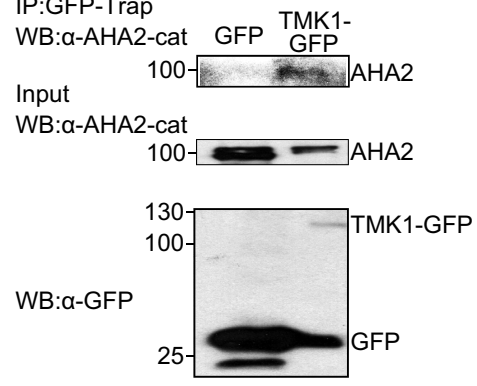

d

MBP-TMK1KD

MBP

GST-AHA1-C

GST

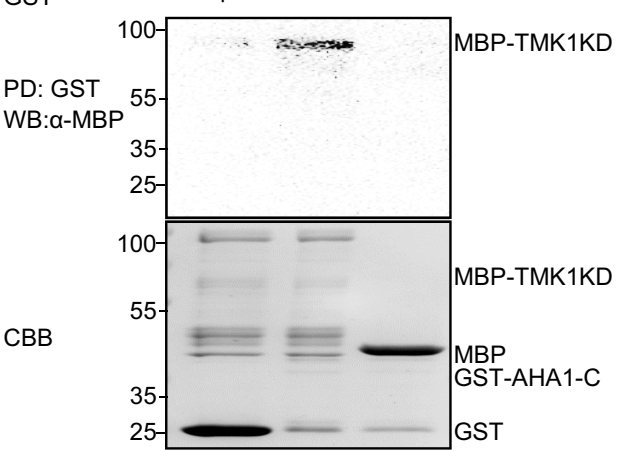

maltose-binding protein (MBP)-TMK1KD or MBP proteins were incubated with glutathione bead-bound glutathione-S-transferase (GST)-AHA2-C or GST

(Pull-down:GST), and the beads were collected and washed for Western blotting of immunoprecipitated proteins with $\alpha$-MBP antibody (left). The input GST-AHA2-C, MBP-TMK1KD, MBP, and GST proteins were detected by Coomassie brilliant blue staining (CBB). e, Representative confocal images of Arabidopsis protoplasts expressing TMK1-mCherry (FRET acceptor) and AHA1-GFP (FRET donor) used for FRET analysis. Shown are images collected before $(-25 \mathrm{sec})$ and after $(+150 \mathrm{sec})$ auxin treatment for three channels: Donor (excitation: $488 \mathrm{~nm}$, emission: 498-551 nm), FRET (excitation: $488 \mathrm{~nm}$, emission:600-670) and acceptor (excitation: $561 \mathrm{~nm}$, emission: $600-670 \mathrm{~nm}$ ). These images are used for FRET efficiency analysis shown in Fig. 1e. To avoid autofluorescence from chorophylls, only the PM region (dotted lines) away from chloroplasts was selected for FRET analysis. Scale bar, $10 \mu \mathrm{m}$. 3 independent analyses were conducted with similar results.

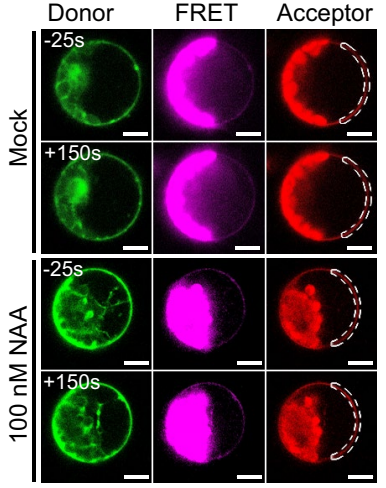

transgenic plants is shown (Bottom).d, TMK1's cytoplasmic kinase domain

(KD) interacts with AHA2's C-terminal domain in vitro. E. coli-expressed
Extended Data Fig. 1 | TMK interacts with AHAs in planta and in vitro. peptides from control pTMK1::GFP seedlings. b, TMK4 associates with AHA1 in transgenic plants. The membrane proteins from 4-week-old 35S::GFP only and 35S::GFP-AHA1 plants were immunoprecipitated with $\alpha$-GFP-Trap antibody and 35S::GFP and $p T M K 1:: T M K 1-G F P /$ tmk1-1/4-1 transgenic plants were immunoprecipitated with $\alpha$-GFP-Trap antibody and analysed with Western blots a, Summary of LC-MS/MS analysis of AHAs associated with TMK1-GFP. The number of unique AHA peptides identified in the immunoprecipitates from pTMK1::TMK1-GFP transgenic seedlings is shown. IP-MS did not identify any AHA analysed with Western blots using an $\alpha$-TMK4 antibody (Top). The express b

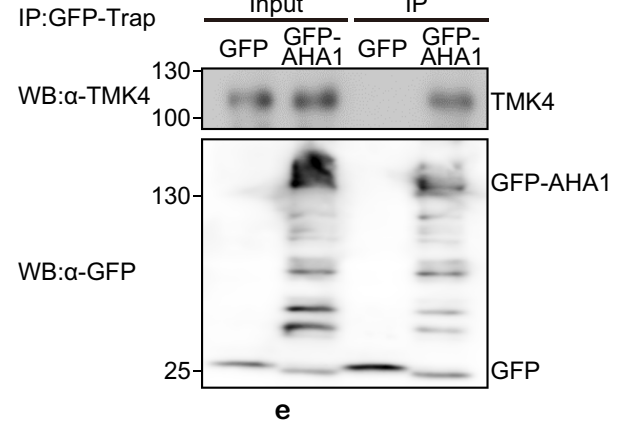


a

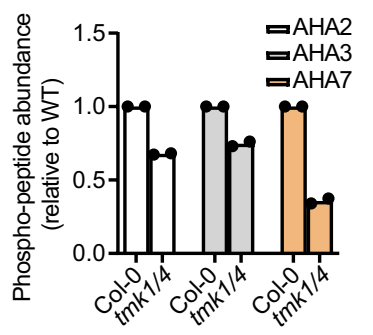

C
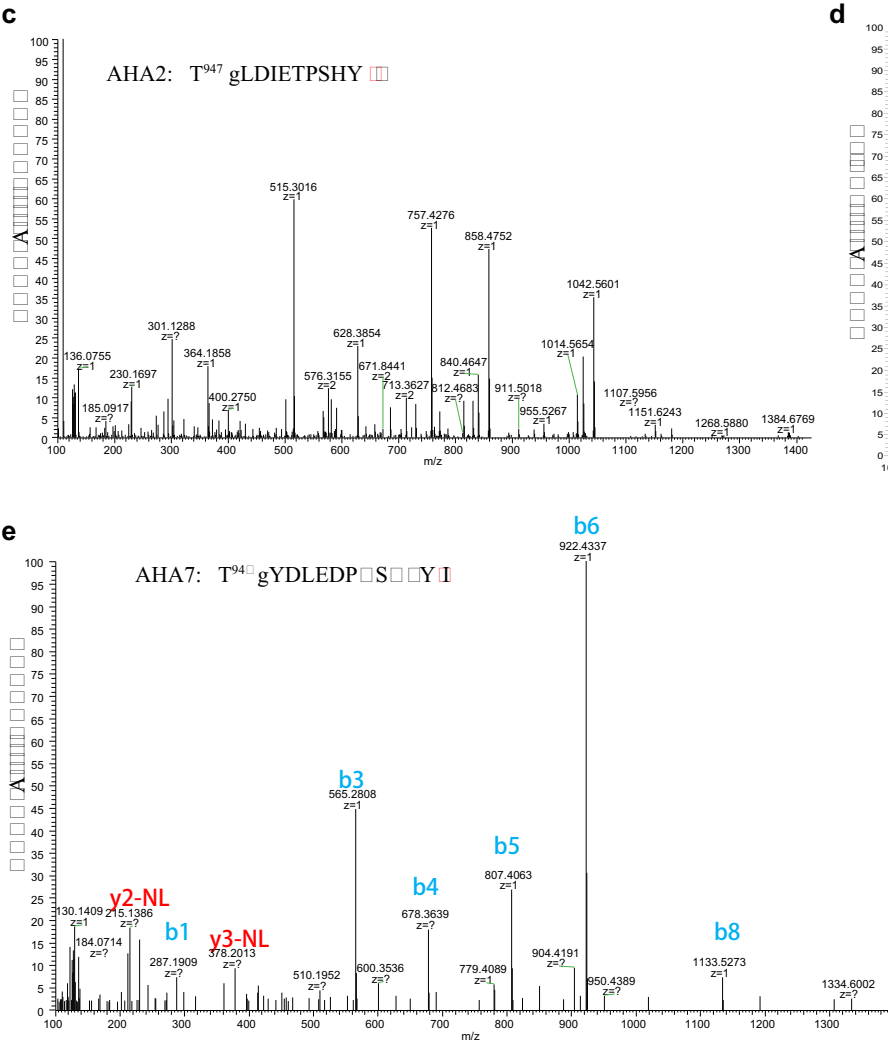

h

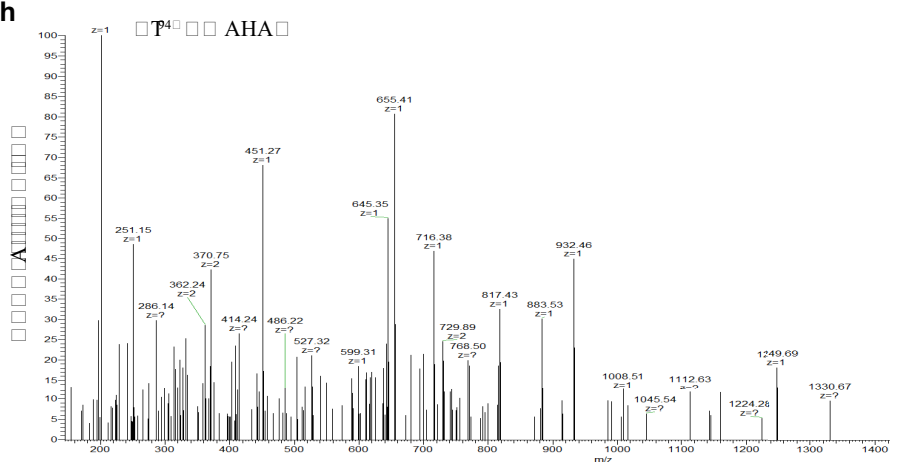

b

d

\begin{tabular}{|c|c|c|c|c|c|c|c|c|c|c|c|}
\hline Annotated Sequence & $\begin{array}{l}\text { Master Protein } \\
\text { Accession }\end{array}$ & $\begin{array}{l}\text { Difference } \\
\text { tmk1/4 / WT }\end{array}$ & $\begin{array}{l}\text { Student's } \\
\text { T-test } \\
\text { p-value }\end{array}$ & $\begin{array}{r}\text { Student's } \\
\text { T-test q- } \\
\text { value }\end{array}$ & PEP & Score & $\begin{array}{l}\text { Delta } \\
\text { score }\end{array}$ & $\begin{array}{l}\text { Score for } \\
\text { localizatio } \\
n\end{array}$ & $\begin{array}{r}\text { Mass } \\
\text { error } \\
{[\mathrm{ppm}]}\end{array}$ & Intensity $\mathrm{F}$ & Position \\
\hline GLDIDTAGHHYT(1)V & $\begin{array}{l}\text { AT2G18960.1 } \\
\text { AHA1 }\end{array}$ & 0.904 & 0.175 & 0.301 & 4.190E-28 & 212.270 & $0 \quad 202.020$ & 212.270 & 0.082 & $1.851 \mathrm{E}+09$ & 948 \\
\hline GLDIETPSHYT(1)V & $\begin{array}{l}\text { AT4G30190.2 } \\
\text { AHA2 }\end{array}$ & 0.678 & 0.002 & 0.016 & 1.820E-10 & 168.300 & 143.080 & o 168.300 & -0.394 & $7.099 \mathrm{E}+09$ & 947 \\
\hline GLDIETAGHYT(1)V & AHA3 & 0.746 & 0.044 & 0.044 & $8.820 \mathrm{E}-05$ & 104.790 & 87.469 & 9104.790 & 0.681 & $1.056 \mathrm{E}+07$ & 948 \\
\hline GYDLEDPNS(0.01)NNY(0.077)T(0.913)I & $\begin{array}{l}\text { AT3G60330.2 } \\
\text { AHA7 } \\
\text { AT3G42640.1 }\end{array}$ & 0.357 & 0.007 & 0.000 & 4.280E-05 & 86.028 & B 74.112 & 86.028 & 0.404 & $3.997 E+06$ & 960 \\
\hline GLDIDTIQQHY $(0.038) \mathrm{T}(0.962) \mathrm{V}$ & $\begin{array}{l}\text { AHA8 } \\
\text { AT5G62670.1 }\end{array}$ & 1.085 & 0.743 & 0.465 & 1.997E-04 & 90.629 & 64.970 & $0 \quad 86.814$ & 0.037 & $4.349 \mathrm{E}+06$ & 947 \\
\hline GLDIETIQQAY $(0.002) \mathrm{T}(0.998) \mathrm{V}$ & AHA11 & 0.916 & 0.240 & 0.360 & 1.270E-05 & 128.850 & 101.730 & o 128.850 & 0.060 & $2.280 \mathrm{E}+08$ & 955 \\
\hline
\end{tabular}

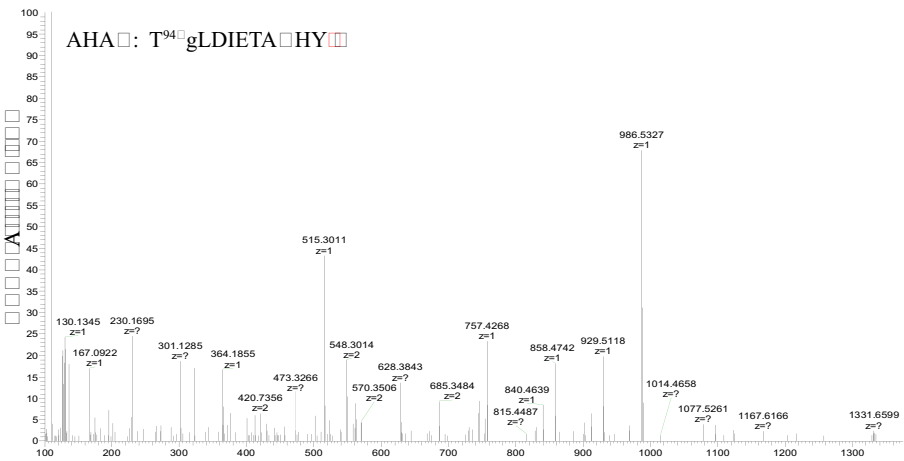

f
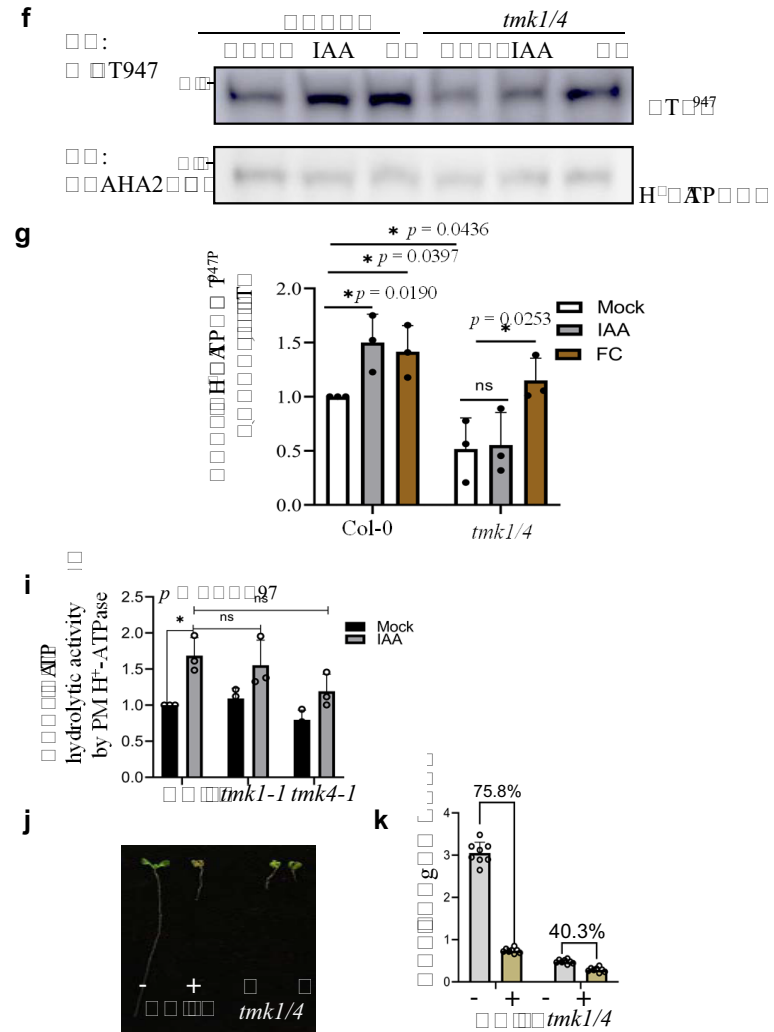

Extended Data Fig. 2 |See next page for caption. 


\section{Article}

Extended Data Fig. 2 TMK1 and TMK4 impact the phosphorylation status of AHAs and the function of the $\mathrm{PM} \mathrm{H}^{+}$-ATPase pump. a, The phosphorylation status of AHAs was changed in the tmk1-1 tmk4-1 (tmk1/4) mutant. The aerial part of 5-days auxin-depleted seedlings was used to prepare membrane proteins for TMT (Tandem mass tag) labelling and mass spectrometry quantification as described in Method. Mass spectrometry analysis showed that the abundance of the peptides containing phosphorylated penultimate threonine from AHA2, AHA3, and AHA7 was significantly decreased in tmk1-1 tmk4-1 (tmk1/4) mutant relative to wild type. Values are means; $n=2$. $\mathbf{b}$, Summary of phosphorylated peptides mass spectrometry information. The C-terminal peptides of AHA1, AHA2, AHA3, AHA7, and AHA11 containing phosphorylated penultimate threonine were identified from mass spectrometry analysis.c-e, High-resolution fragmentation spectra of AHA2 (c), AHA3(d), and AHA11(e) C-terminal peptides containing phosphorylated penultimate threonine are presented.f, The tmk1-1 tmk4-1(tmk1/4) mutant is insensitive to auxin inducing AHA phosphorylation but remains sensitive to fusicoccin. The endogenous auxin-depleted aerial sections of seedlings were incubated with $100 \mathrm{nM}$ IAA for $10 \mathrm{~min}$ or $10 \mu \mathrm{m}$ fusicoccin (FC) for $5 \mathrm{~min}$, respectively. The amounts of AHA proteins and the phosphorylation status of the penultimate $\mathrm{Thr}$ in the $\mathrm{C}$ terminus were determined by immunoblot analysis with anti-AHA ( $\mathrm{H}^{+}$-ATPase) and anti-pThr-947 (pThr 947) antibodies, respectively. $\mathbf{g}$, Quantification of the phosphorylation level of the $\mathrm{H}^{+}$-ATPase. Values are means $\pm S D ; n=3$ independent biological replicates, ${ }^{*} \mathrm{P} \leq 0.05$; $n \mathrm{n}$, no significant, results of two way ANOVA test. h, Fragmentation spectra of peptides containing phosphorylated penultimate threonine of AHA1-C16 synthetical peptide (pT948 of AHA1) (see Fig. 2e). $\mathbf{i}$, Auxin induction of $\mathrm{H}^{+}$-ATPase activity in the aerial parts of wild type, the tmk1-1, and tmk4-1 mutant. Aerial sections of 14-days old seedlings were treated with $10 \mu \mathrm{m} \mathrm{IAA}$ for $30 \mathrm{~min}$ and used for vanadate-sensitive ATP hydrolysis assay by determining the inorganic phosphate released from ATP as described previousl ${ }^{27}$. The values shown are relative ATP hydrolytic activity of indicated samples to that of control $\mathrm{Col}-0$ without auxin treatment. Values are means $\pm \mathrm{SD} ; \mathrm{n}=3 .{ }^{*} \mathrm{P} \leq 0.05$; $\mathrm{ns}$, not significant. The results were analysed by a two-way ANOVA test.j, Lithium tolerance in the tmk1-1 tmk4-1 (tmk1/4) mutant. Wild type (Col-0) and tmk1-1 tmk4-1 (tmk1/4) mutant seedlings were grown on $1 / 2 \mathrm{MS}$ medium with or without $18 \mathrm{mM} \mathrm{LiCl} \mathrm{for} 5$ days. $\mathrm{LiCl}$ treatment caused severe seedling growth retardation and severe chlorosis of the aerial parts in Col-0, whereas the tmk1-1 tmk4-1 (tmk1/4) mutant was tolerant to $\mathrm{LiCl}$, especially in the aerial parts. $\mathbf{k}$, The root length of the seedlings was measured by ImageJ. Values are means $\pm S D, n=8$. The number above the columns indicates the percentage of root growth inhibition induced by $\mathrm{LiCl}$. 
a

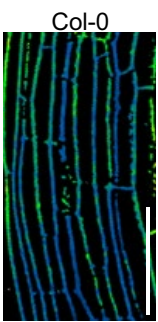

C

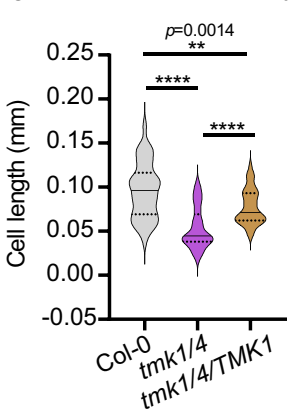

d

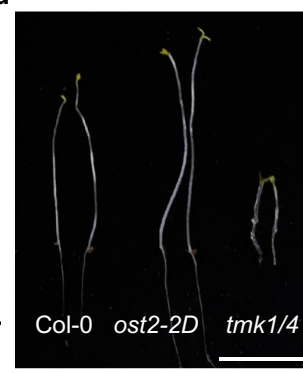

b
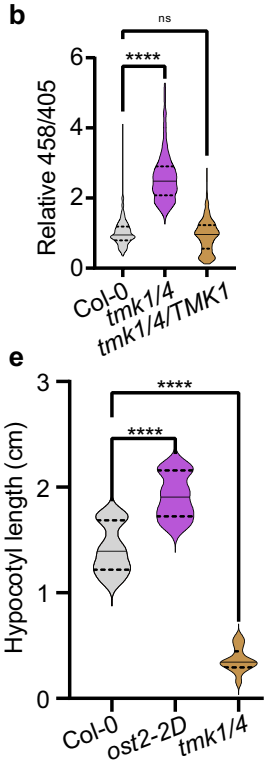

Extended Data Fig. 3 | TMK1 and TMK4 regulate apoplastic pH and

hypocotyl elongation. a, TMK1 ( $p T M K 1:: T M K 1-G F P)$ restored the apoplastic $\mathrm{pH}$ changes in the tmk1-1 tmk4-1 (tmk1/4) mutant. Comparison of the apoplastic $\mathrm{pH}$ in WT, the tmk1-1 tmk4-1 mutant, and the tmk1-1 tmk4-1/TMK1-GFP (tmk1/4) $T M K 1)$ complemented line. Visualized by HPTS staining (a). Y-Axis: the mean 458/405 values of the tmk1-1 tmk4-1 mutant and the TMK1 complemented line relative to wild type (b). 3 independent assays were conducted with similar results. c, Epidermal cell lengths of hypocotyls from two days-old etiolated seedlings were measured using Image J. Hypocotyl epidermal cells in the 100$500 \mu \mathrm{M}$ region after apical hook were measured. $n=41,52$, and 53 for Col- 0 , $t m k 1 / 4$ and $t m k 1 / 4 / T M K 1$. The results were analysed by one Way ANOVA tests in b, c. ${ }^{* * * *} \mathrm{P} \leq 0.0001$. d and e, The tmk1-1 tmk4-1 mutant showed a defect in hypocotyl elongation (c). Hypocotyl lengths of 3 days-old etiolated seedlings were measured by Image J (d). $\mathrm{n}=21,11$, and 14 for Col-0, ost2-2D, and tmk1/4, respectively. The results were analysed by one-way ANOVA tests in b, c, e. Scale bar $=100 \mu \mathrm{M}(\mathbf{a})$, or $1 \mathrm{~cm}(\mathbf{d}) .{ }^{* *} \mathrm{P} \leq 0.01,{ }^{* * * *} \mathrm{P} \leq 0.0001$; ns, not significant. 


\section{Article}
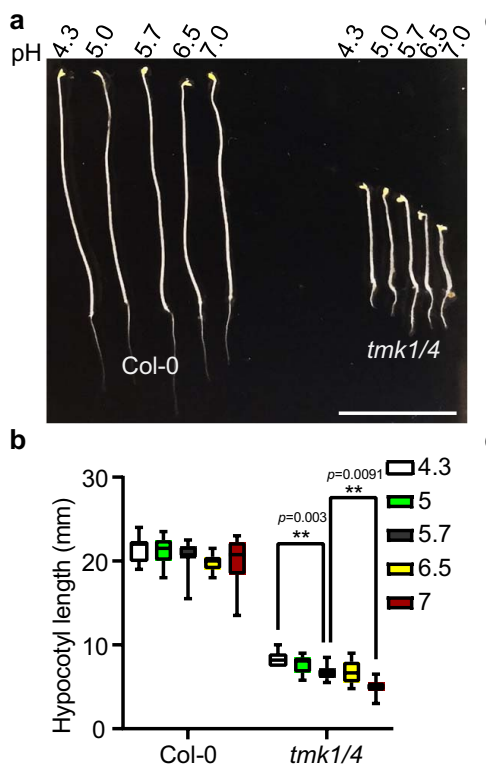
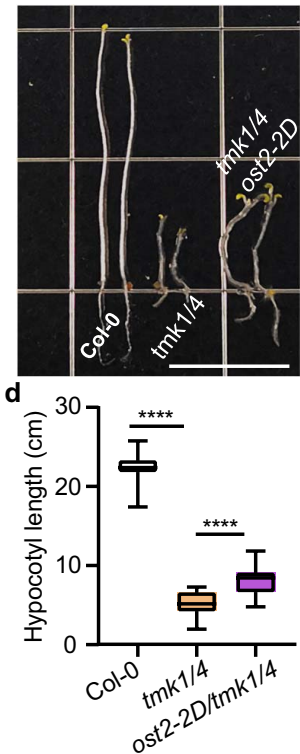

Extended Data. Fig. 4 | Acidic environments and activation of the PM $\mathrm{H}^{+}$-ATPase pump partially restored hypocotyl elongation defect in $\mathrm{tmk1-1}$ tmk4-1. $a$ and b, Low $\mathrm{pH}$ in the medium was able to partially restore the tmk1-1 tmk4-1 (tmk1/4) defect in hypocotyl elongation. Seedlings were grown on $1 / 2 \mathrm{MS}$ medium with indicated $\mathrm{pH}(\mathbf{a})$, and hypocotyl lengths were measured by ImageJ (b). The box plot centre lines represent median; box limits indicate the $25^{\text {th }}$ and $75^{\text {th }}$ percentiles; and whisker extend $1.5 x I Q R$ from the $25^{\text {th }}$ and $75^{\text {th }}$ percentiles ( $n=15$ hypocotyls per line). $c$ and d, ost $2-2 D$ mutation partially restored the hypocotyl elongation defect of $t m k 1-1 \mathrm{tmk} 4-1$ mutant. Seedlings were grown on a $1 / 2$ MS medium for 4 days, and hypocotyl lengths were measured by ImageJ. The box plot centre lines represent median; box limits indicate the $25^{\text {th }}$ and $75^{\text {th }}$ percentiles; and whisker extend 1.5xIQR from the $25^{\text {th }}$ and $75^{\text {th }}$ percentiles $(n=36$ for Col- $0, \mathrm{n}=24$ for $t m k 1 / 4$ and $\mathrm{n}=21$ for 0 st $2-2 D / t m k 1 / 4$ ). Results were analysed by one-way ANOVA tests in b, d. Scale bar $=10 \mathrm{~mm} .{ }^{* *} p \leq 0.01,{ }^{* * * *} p \leq 0.0001$. 


\section{Reporting Summary}

Nature Portfolio wishes to improve the reproducibility of the work that we publish. This form provides structure for consistency and transparency in reporting. For further information on Nature Portfolio policies, see our Editorial Policies and the Editorial Policy Checklist.

\section{Statistics}

For all statistical analyses, confirm that the following items are present in the figure legend, table legend, main text, or Methods section.

n/a Confirmed

$\square$ The exact sample size $(n)$ for each experimental group/condition, given as a discrete number and unit of measurement

$\square \bigotimes$ A statement on whether measurements were taken from distinct samples or whether the same sample was measured repeatedly

$\square$ The statistical test(s) used AND whether they are one- or two-sided

$\square$ Only common tests should be described solely by name; describe more complex techniques in the Methods section.

Х $\square$ A description of all covariates tested

$\square$ \A description of any assumptions or corrections, such as tests of normality and adjustment for multiple comparisons

$\square$ A full description of the statistical parameters including central tendency (e.g. means) or other basic estimates (e.g. regression coefficient)

$\triangle$ AND variation (e.g. standard deviation) or associated estimates of uncertainty (e.g. confidence intervals)

$\square$ For null hypothesis testing, the test statistic (e.g. $F, t, r$ ) with confidence intervals, effect sizes, degrees of freedom and $P$ value noted

Give P values as exact values whenever suitable.

\ $\square$ For Bayesian analysis, information on the choice of priors and Markov chain Monte Carlo settings

Х $\square$ For hierarchical and complex designs, identification of the appropriate level for tests and full reporting of outcomes

Х $\square$ Estimates of effect sizes (e.g. Cohen's $d$, Pearson's $r$ ), indicating how they were calculated

Our web collection on statistics for biologists contains articles on many of the points above.

\section{Software and code}

Policy information about availability of computer code

Data collection Proteomic data was collected by Orbitrap Fusion (Thermo Fisher Scientific, Watham, MA), by software Thermo Xcalibur 3.0.63

Data analysis 1: Arabidopsis root and hypocotyl tissue and cell length are all measured by ImageJ (JAVA 1.8.0_172). 2: FRET analyzer, an ImagJ plug-in, was used to analyze FRET signal. 3: The ratio metric image was analyzed and quantified by Fiji (JAVA 1.8.0_172), using a macro language, which was described in the manuscript. 4:LC-MS data were analyzed with Maxquant (version 1.6.2.6) with Andromeda search engine.

For manuscripts utilizing custom algorithms or software that are central to the research but not yet described in published literature, software must be made available to editors and reviewers. We strongly encourage code deposition in a community repository (e.g. GitHub). See the Nature Portfolio guidelines for submitting code \& software for further information.

\section{Data}

Policy information about availability of data

All manuscripts must include a data availability statement. This statement should provide the following information, where applicable:

- Accession codes, unique identifiers, or web links for publicly available datasets

- A description of any restrictions on data availability

- For clinical datasets or third party data, please ensure that the statement adheres to our policy

Code and Data availability statement was included in Methods Section. Mass spectrometry raw data associated with Figure 2e, extended Data Figure 2a,b are available at the MassIVE under accession number: MSV000087822. Source Data (gel and graphs) are provided with manuscript. The data supporting the findings in this study are available and described within the manuscript and extend data information file. 


\section{Field-specific reporting}

Please select the one below that is the best fit for your research. If you are not sure, read the appropriate sections before making your selection. $\bigotimes$ Life sciences $\quad \square$ Behavioural \& social sciences $\quad \square$ Ecological, evolutionary \& environmental sciences

For a reference copy of the document with all sections, see nature.com/documents/nr-reporting-summary-flat.pdf

\section{Life sciences study design}

All studies must disclose on these points even when the disclosure is negative.

Sample size

Sample size calculation was not performed. We determined the number of samples in each experiment as commonly accepted standards in the field. 1: Western blot. All the western blot assays were repeat 3-4 times and the intensity peak of the target bands were measured by ImageJ. 2: Quantitative analysis of hypocotyl elongation zone apoplastic $\mathrm{pH}$ value. Each data set was from the measurement of 10-20 etiolated hypocotyls of different genotypes. 3: MS to identified in vitro peptides phosphorylation. Two biological repeats were included, in each biological repeat, 3 independent technique repeats were included. 4: TMT-label based phosphoproteomics MS. 0.45 mg proteins of each sample were applied to TMT-labeled and ms analysis. 2 independent biological repeats were performed for this assay. 1-5 mg proteins were applied to IP-MS. 5: For FRET assay. The data was collected and analyzed from 10 individual cells. The experiment was repeated 3 times.

Data exclusions No data were excluded from this study.

$\begin{array}{lll}\text { Replication } & \text { The data in thsi paper is highly replicable, as the companion paper conduct several experiments independently used same materials produced }\end{array}$ same results. All the measures in this study were conducted in 2-4 times biology repeats, which start from germination of the seedlings. Each set of the data were collected and analyzed independently.

Randomization The study does not involved work that required random allocation. The sample were allocated into experimental groups based on their genotypes, for instance, by wild type or specific gene mutations. The randomization was not applied in this study.

Blinding

No double blinding is applied in this study. For this current study, blinding is not relevant.

\section{Reporting for specific materials, systems and methods}

We require information from authors about some types of materials, experimental systems and methods used in many studies. Here, indicate whether each material, system or method listed is relevant to your study. If you are not sure if a list item applies to your research, read the appropriate section before selecting a response.

Materials \& experimental systems

n/a Involved in the study

$\square$ Antibodies

$\bigotimes \square$ Eukaryotic cell lines

\ $\square$ Palaeontology and archaeology

Х $\square$ Animals and other organisms

\ $\square$ Human research participants

$\bigotimes \square$ Clinical data

$\bigotimes \square$ Dual use research of concern

\begin{tabular}{l|l} 
Methods \\
\hline n/a Involved in the study \\
$\square$ \\
$\square$ ChIP-seq \\
$\square$ \\
$\square$ \\
$\square$ Flow cytometry
\end{tabular}

\section{Antibodies}

Antibodies used

1: The anti-HA (Invitrogen, \# 26183, 1:2000 dilution), GFP (Chromotek, \#3h9, 1:1000 dilution), Myc (sinobiological, \#100029-MM08, 1:1000 dilution), GST (Santa Cruz, \#sc-138, 1:1000 dilution) and MBP (Invitrogen, PA1-989, 1:1000 dilution ) antibodies that were used in this study are all commercial available with the validations.

2: PT947 AHA antibody was described in manuscript, which was generated from rabbit (1:5000 dilution).

Validation

Validation statements of commercial primary antibodies are available from manufacturers. $\alpha$-GFP (https://www.chromotek.com/ fileadmin/content/PDFs/Data_Sheets/3h9_Datasheet_GFP_antibody_3H9.pdf), $\alpha$-HA-HRP (https://www.thermofisher.com/order/ genome-database/dataSheetPdf producttype=antibody\&productsubtype=antibody_primary\&productld=26183-HRP\&version=133), $\alpha$-myc (http://www.sinobiologicalcdn.com/reagent/100029-MM08.pdf). $\alpha$-MBP (https://www.thermofisher.com/order/genomedatabase/dataSheetPdf?producttype=antibody\&productsubtype=antibody_primary\&productld=PA1-989\&version=133). $\alpha$-GST (https://datasheets.scbt.com/sc-138.pdf). pT947 antibody was validated as reference: doi:10.1093/pcp/pcq078. 